\title{
gु \\ All-optical magnetization reversal by circularly polarized laser pulses: Experiment and multiscale modeling
}

\author{
K. Vahaplar, ${ }^{1}$ A. M. Kalashnikova,,${ }^{2}{ }^{*}$ A. V. Kimel, ${ }^{1, \dagger}$ S. Gerlach, ${ }^{3}$ D. Hinzke, ${ }^{3}$ U. Nowak, ${ }^{3}$ R. Chantrell, ${ }^{4}$ A. Tsukamoto, ${ }^{5,6}$ \\ A. Itoh, ${ }^{5}$ A. Kirilyuk, ${ }^{1}$ and Th. Rasing ${ }^{1}$ \\ ${ }^{1}$ Radboud University Nijmegen, Institute for Molecules and Materials, Heyendaalseweg 135, 6525 AJ, Nijmegen, The Netherlands \\ ${ }^{2}$ Ioffe Physical-Technical Institute of RAS, Politekhnicheskaya 26, 194021 St. Petersburg, Russia \\ ${ }^{3}$ Universität Konstanz, Fachbereich Physik, Universitatssträße 10, D-78457 Konstanz, Germany \\ ${ }^{4}$ Department of Physics, University of York, Heslington, York YO10 5DD, United Kingdom \\ ${ }^{5}$ College of Science and Technology, Nihon University, 7-24-1 Funabashi, Chiba, Japan \\ ${ }^{6}$ PRESTO, Japan Science and Technology Agency, 4-1-8 Honcho Kawaguchi, Saitama, Japan \\ (Received 15 July 2011; revised manuscript received 22 December 2011; published 6 March 2012)
}

\begin{abstract}
We present results of detailed experimental and theoretical studies of all-optical magnetization reversal by single circularly-polarized laser pulses in ferrimagnetic rare earth-transition metal (RE-TM) alloys $\mathrm{Gd}_{x} \mathrm{Fe}_{90-x} \mathrm{Co}_{10}$ $(20 \%<x<28 \%)$. Using single-shot time-resolved magneto-optical microscopy and multiscale simulations, we identified and described the unconventional path followed by the magnetization during the reversal process. This reversal does not involve precessional motion of magnetization but is governed by the longitudinal relaxation and thus has a linear character. We demonstrate that this all-optically driven linear reversal can be modeled as a result of a two-fold impact of the laser pulse on the medium. First, due to absorption of the light and ultrafast laser-induced heating, the medium is brought to a highly nonequilibrium state. Simultaneously, due to the ultrafast inverse Faraday effect the circularly polarized laser pulse acts as an effective magnetic field of the amplitude up to $\sim 20 \mathrm{~T}$. We show that the polarization-dependent reversal triggered by the circularly polarized light is feasible only in a narrow range (below 10\%) of laser fluences. The duration of the laser pulse required for the reversal can be varied from $\sim 40$ fs up to at least $\sim 1700 \mathrm{fs}$. We also investigate experimentally the role of the ferrimagnetic properties of GdFeCo in the all-optical reversal. In particular, the optimal conditions for the all-optical reversal are achieved just below the ferrimagnetic compensation temperature, where the magnetic information can be all-optically written by a laser pulse of minimal fluence and read out within just $30 \mathrm{ps}$. We argue that this is the fastest write-read event demonstrated for magnetic recording so far.
\end{abstract}

DOI: 10.1103/PhysRevB.85.104402

PACS number(s): 75.40.Gb, 75.60.Jk, 85.70.Li

\section{INTRODUCTION}

The problem of very fast switching the magnetization between its metastable states emerged recently as one of the most exciting topics in magnetism. ${ }^{1}$ The fastest conventional way to reverse magnetization is based on a precessional motion of the latter in an orthogonal external magnetic field. A realistic switching time, which can be achieved in such a process, is $\sim 100$ ps and is determined by the strength and duration of the magnetic field pulse. ${ }^{2}$ On such a time scale the magnetization dynamics can be fully described in terms of adiabatic and macrospin approximations. However, it has been discovered that reduction of the magnetic field pulse duration below 2.3 ps may result in a stochastic magnetization switching, ${ }^{3}$ showing that the macrospin approximation fails to describe the process. ${ }^{4}$ Furthermore, the dynamics of spins on such a short timescale is not fully understood so far.

One of the most intriguing alternatives to magnetic field-induced magnetization switching is making use of a subpicosecond laser pulse. Already the first observation of subpicosecond demagnetization of a thin Ni film subjected to a 60 -fs laser pulse ${ }^{5}$ suggested that such a pulse represents a powerful stimulus that is able to cause ultrafast changes in the magnetic state of matter. At the same time, this and the following studies of the various effects occurring in magnetic media under the action of ultrashort laser pulses rose a number of important fundamental questions. ${ }^{6-11}$ For instance, let us consider a magnetic medium subjected to a strong external perturbation, the duration of which is comparable to or even shorter than the characteristic times of equilibration between different reservoirs of energy and angular momentum. Such a perturbation brings a magnetic medium into a strongly nonequilibrium state, where a conventional description of magnetic phenomena in terms of thermodynamics and adiabatic approximations is no longer valid. Therefore, from the point of view of theoretical physics, there is a need for developing novel approaches and approximations capable of an adequate description of the ultrafast spin dynamics at extremely short time scales. From the experimental point of view, studies of such a dynamics also require techniques that combine subpicosecond time resolution with a sensitivity to changes of the magnetic ordering. Consequently, attempts to understand ultrafast light-induced magnetization dynamics have yielded a burst in the development of various experimental ${ }^{11-17}$ and theoretical ${ }^{18-23}$ approaches (for a recent review, see Ref. 24).

Recently, it has been demonstrated that a 40-fs circularly polarized pulse can reverse the magnetization in a ferrimagnetic metallic film. ${ }^{25}$ Both the experimental investigation of the details of this all-optical helicity-dependent magnetization reversal and its theoretical description constitute a number of challenging issues. In our preceding work, ${ }^{26}$ we have shown that this all-optical reversal of magnetization proceeds via an unusual route where an ultrashort laser pulse first brings the medium into a strongly nonequilibrium state with no net magnetization. It was suggested that in this state, 
a circularly polarized pulse acting as an effective magnetic field steers the linear ${ }^{27}$ magnetization reversal. Nevertheless, there are still many open questions related to the all-optical magnetization reversal with circularly polarized light. First, there is a noticeable discrepancy between switching times calculated for $10^{6}$ exchange coupled spins $(30 \mathrm{~nm})^{3}$ and those observed in the experiment for micrometer-sized domains. ${ }^{26}$ In order to describe the behavior of systems of realistic sizes, it is important to develop a multiscale modeling of the ultrafast spin dynamics. Moreover, all the experimental studies performed so far have been employing only subpicosecond laser pulses, ${ }^{25,26,28}$ while the simulations suggest that the elongation of the laser pulse to the picosecond range can ease the reversal process. Therefore it is important to investigate how the all-optical magnetization reversal depends on the duration of the laser pulse. Finally, we already have shown that the speed of the all-optical reversal is strongly affected by the ferrimagnetic properties of GdFeCo alloys. ${ }^{26}$ How does the efficiency of the reversal depend on the magnetic properties of the alloy? This question becomes especially interesting in the light of unexpected spin dynamics, which has been recently unveiled in a ferrimagnetic $\mathrm{GdFeCo}$ alloy. ${ }^{29}$

To answer all these questions, we performed comprehensive studies of all-optical reversal of magnetization by subpicoand picosecond circularly polarized laser pulses in ferrimagnetic metallic rare earth-transition metal (RE-TM) alloys $\mathrm{Gd}_{x} \mathrm{Fe}_{90-x} \mathrm{Co}_{10}$. In order to investigate the helicity-dependent reversal process experimentally, we developed a single-shot time-resolved magneto-optical microscopy approach ${ }^{26}$ that combined subpicosecond temporal with (sub)micrometer spatial resolutions. This enabled us to study transient and steady states occurring in samples as a result of the action of a single laser pulse with various fluences, polarizations, and durations. In order to describe the dynamics of a spin system subjected to such an intensive and short laser pulses, we employed a recently developed multiscale calculation technique, ${ }^{19}$ which we extended to magnetic films of a size of up to $10 \times 10 \mu \mathrm{m}^{2}$. Using these techniques, we investigated the mechanism of the all-optical reversal in details.

In particular, we have showed that the actual all-optical helicity-dependent reversal occurs at a timescale of just several picoseconds and has a linear character, while the rest of the process is governed by the relaxation of the medium to an equilibrium. In addition to the results reported in our preceding work, ${ }^{26}$ we demonstrate that the reversal can be observed in a rather wide range of laser pulse durations. Furthermore, these experimental results along with the extended calculations suggest that there exist both lower and upper limits for the laser pulse durations. The upper limit is due to the fact that a metallic magnet excited by a too long laser pulse cannot reach the strongly nonequilibrium state with no net magnetization. The lower limit, the existence of which has still to be experimentally verified, is related to the fact that the duration of the laser-induced effective field duration becomes too short compared to the electron equilibration times. Our experimental studies of the all-optical reversal in various $\mathrm{GdFeCo}$ alloys have revealed that the compensation temperature of the samples has a significant effect on several parameters. In particular, we demonstrate that not only the switching speed, as we reported earlier, ${ }^{26}$ but also the laser pump fluence required for the switching depends prominently on the proximity near the compensation point of the samples. These observations highlight the role of the particular magnetic structure in the response of a spin system to the ultrashort laser pulse excitation. Moreover, these results motivate further development of theoretical methods for a realistic description of the ultrafast spin dynamics in complex magnetic media.

The paper is organized as follows. The structure, magnetic, and magneto-optical properties of the GdFeCo alloys are described in Sec. II. In Sec. III, we describe the details of the single-shot time-resolved magneto-optical microscopy technique and the multiscale calculations. This is followed by a discussion and comparison between the experimental and the modeling results. In particular, in Secs. IV A and IV B, we present an extended discussion of the unusual linear route for the magnetization reversal, identified in Ref. 26. Then we show how the all-optical reversal changes as a function of the laser pulse duration (see Sec. IV C) and the ferrimagnetic properties of the samples (see Sec. IV D). Finally, we present an outlook (see Sec. V), where we specify the most important open questions left.

\section{SAMPLES}

The magnetic materials studied in this work are $20-\mathrm{nm}$ thick films of the ferrimagnetic rare earth-transition metal (RE-TM) amorphous alloy $\mathrm{Gd}_{x} \mathrm{Fe}_{90 . .91-x} \mathrm{Co}_{9 . .10}(14<x<$ 30). The samples were grown by magnetron sputtering in the following multilayer structure: glass $|\operatorname{AlTi}(10 \mathrm{~nm})| \operatorname{SiN}(5$ $\mathrm{nm})|\mathrm{GdFeCo}(20 \mathrm{~nm})| \operatorname{SiN}(60 \mathrm{~nm})$. The AlTi layer serves as a heat sink and the SiN as buffer and capping layers. The latter one also serves as an antireflection coating.

$\mathrm{Gd}_{x} \mathrm{Fe}_{100-x-y} \mathrm{Co}_{y}$ alloys are ferrimagnets, where the Fe and Gd sublattices are coupled antiferromagnetically, while the Co magnetic moments are parallel to those of iron. These alloys are widely used in magneto-optical recording and known for their strong magneto-optical effects. ${ }^{30,31}$ Depending on RE ions concentration RE-TM alloys can exhibit magnetization $\left(T_{\mathrm{M}}\right)$ and angular momentum $\left(T_{\mathrm{A}}\right)$ compensation temperatures, where the magnetizations (angular momenta) of the RE and TM sublattices are equivalent and, consequently, the net magnetization (angular momentum) is zero. For the case of Gdcontaining alloys, the magnetization compensation point may be expected below the Curie point $T_{\mathrm{C}}$ if $x=20 \%, \ldots, 30 \%$ (see Ref. 32). The angular momentum compensation point is typically $\sim 50 \mathrm{~K}$ above $T_{\mathrm{M}} \cdot{ }^{33}$ Along with $T_{\mathrm{M}}$, a number of other magnetic properties, such as magnetic anisotropy and coercive field, are defined by the $\mathrm{Gd}$ concentration. ${ }^{32}$ The Co ions, substituting for $0 \%, \ldots, 30 \%$ of $\mathrm{Fe}$ ions in $\mathrm{RE}_{x} \mathrm{Fe}_{100-x-y} \mathrm{Co}_{y}$ alloys, on the one hand, enhance the Curie temperature $T_{\mathrm{C}}$ of the alloys and, on the other hand, increase their magneto-optical susceptibility. ${ }^{34,35}$

We investigated the magnetic and magneto-optical properties of the $\mathrm{Gd}_{x} \mathrm{Fe}_{\sim 90-x} \mathrm{Co}_{\sim 10}$ samples with $\mathrm{Gd}$ ions concentration $x$ in the range from $14 \%$ to $30 \%$. For this purpose, the magneto-optical Faraday effect in the samples was measured at a photon energy of $1.52 \mathrm{eV}$ as a function of sample temperature and magnetic field, which was applied along the normal of the sample. The Curie temperature $\left(T_{\mathrm{C}}\right)$ for all studied samples was found to be $T_{\mathrm{C}} \approx 550 \mathrm{~K}$. We found that 


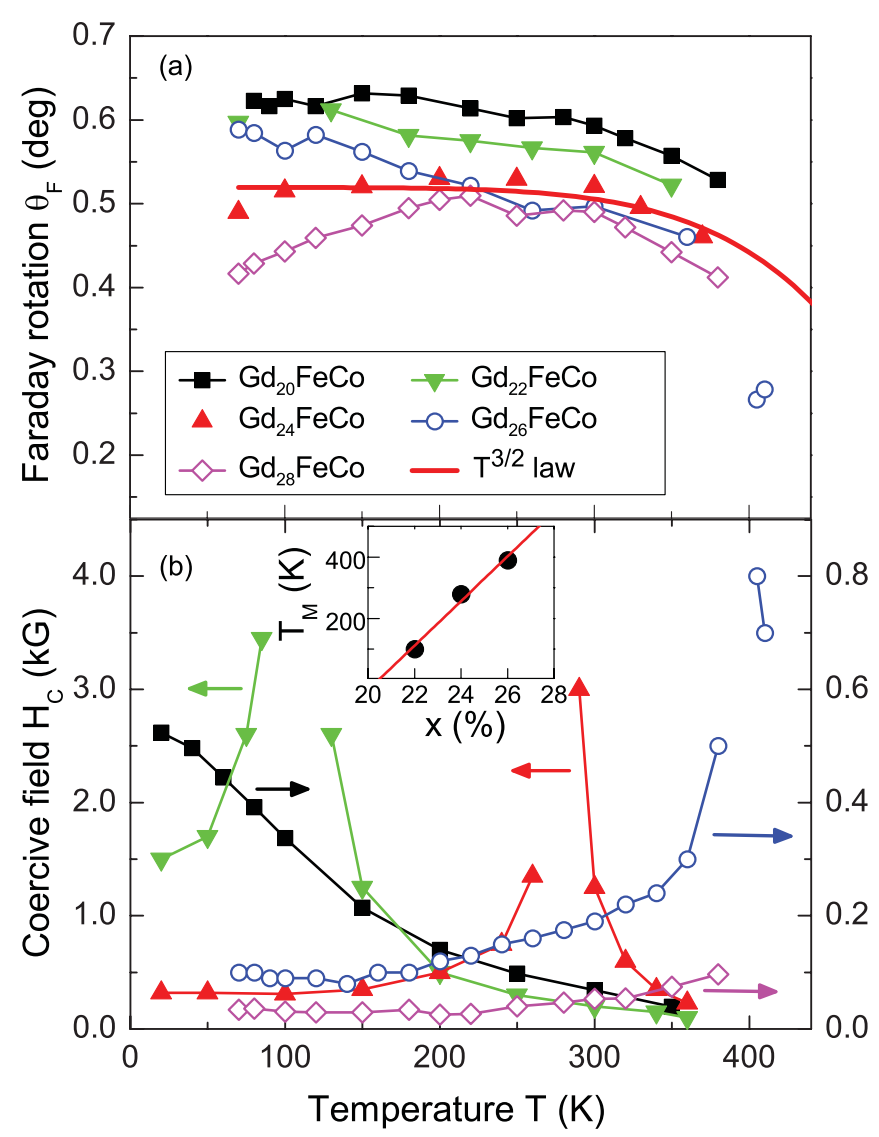

FIG. 1. (Color online) (a) Amplitude of the Faraday rotation $\theta_{\mathrm{F}}$ and (b) coercive field $H_{\mathrm{c}}$ vs temperature $T$ for the samples $\mathrm{Gd}_{x} \mathrm{FeCo}$, where $x=20 \%, 22 \%, 24 \%, 26 \%$, and $28 \%$, as obtained from the field dependence of the Faraday rotation measured at different temperatures for a photon energy of $1.52 \mathrm{eV}$ (lines and symbols). Thick line in (a) shows the $T^{3 / 2}$ law for the magnetization of the FeCo sublattice. The inset in (b) shows the dependence of the magnetization compensation temperature $T_{\mathrm{M}}$ on the concentration of the $\mathrm{Gd}$ ions. The line is a linear fit $\left(T_{\mathrm{M}}=-1483+72.5 x\right)$.

GdFeCo films exhibited perpendicular magnetic anisotropy in the range of $\mathrm{Gd}$ concentrations $20 \%<x<28 \%$. In the rest of the paper, we will discuss the results obtained for the alloys $\mathrm{Gd}_{20} \mathrm{Fe}_{70} \mathrm{Co}_{10}, \mathrm{Gd}_{22} \mathrm{Fe}_{68.2} \mathrm{Co}_{9.8}, \mathrm{Gd}_{24} \mathrm{Fe}_{66.5} \mathrm{Co}_{9.5}$, $\mathrm{Gd}_{26} \mathrm{Fe}_{64.7} \mathrm{Co}_{9.3}$, and $\mathrm{Gd}_{28} \mathrm{Fe}_{63} \mathrm{Co}_{9}$. As the $\mathrm{Fe}$ and Co contents do not vary significantly from one sample to another, in the rest of the paper we omit in the sample names the subscripts for the $\mathrm{Fe}$ and $\mathrm{Co}$ concentration. Thus these samples will be referred as $\mathrm{Gd}_{20} \mathrm{FeCo} . . . \mathrm{Gd}_{28} \mathrm{FeCo}$, respectively.

Figure 1(a) shows the temperature dependence of the Faraday rotation of these samples at saturation. The value of the Faraday rotation is of the order of $1^{\circ}$ (i.e., $5 \times 10^{5} \mathrm{deg} / \mathrm{cm}$ ). This value decreases with an increase of the Gd concentration $x$. In the studied range of temperatures $T=10-420 \mathrm{~K}$ the Faraday rotation exhibits a weak temperature dependence. It is conventionally assumed that in the visible spectral range the magneto-optical signal is dominated by contributions originating from the iron sublattice. Therefore the change of the Faraday rotation with temperature, shown in Fig. 1(a), reflects the magnetization changes of the iron sublattice with temperature.
For characterizing the strength of magneto-optical and optomagnetic ${ }^{24,36}$ interactions one needs to know the magnetooptical susceptibility $\beta$, which determines the correlation between the optical response of a medium and the magnetization of the latter. The Faraday rotation angle $\theta_{\mathrm{F}}$ is determined by the off-diagonal component $i \varepsilon_{x y}$ of the dielectric tensor as

$$
\theta_{\mathrm{F}}=\frac{\pi d}{\lambda} \frac{\varepsilon_{x y}}{n}=\frac{\pi d}{\lambda} \frac{\beta M_{0}}{n},
$$

where $\lambda$ is the wavelength, $d$ is the GdFeCo thickness, and $n$ is the refraction index, determining the refraction of light. The magneto-optical susceptibility $\beta$ is related to $\varepsilon_{x y}$ as $\varepsilon_{x y}=\beta M_{0} . M_{0}$ here is the magnetization of the $\mathrm{Fe}$ sublattice. Assuming the magnetic moments of $\mathrm{Fe}$ ions to be $2.217 \mu_{\mathrm{B}} /$ at. (the value for a pure iron), yields the magnetization of $\mathrm{Fe}$ sublattice of order of $7 \times 10^{5} \mathrm{~A} / \mathrm{m}$. For the case of REFeCo alloy, this is, obviously, overestimated. For instance, the net magnetization of $\mathrm{GdFeCo}$, containing $23 \%$ Gd ions and $\sim 70 \% \mathrm{Fe}$ ions is of order of $0.8-1 \times 10^{5} \mathrm{~A} / \mathrm{m}$ at low temperatures, ${ }^{32}$ at which $M_{\mathrm{RE}} / M_{\mathrm{FE}} \sim 2$. Thus the iron sublattice magnetization should be approximately $0.8-1 \times$ $10^{5} \mathrm{~A} / \mathrm{m}$. Since the iron sublattice magnetization does not decrease much in the range of $T=10-400 \mathrm{~K}$, we take these values as an estimate for $M_{0}$ in this temperature range. Therefore the magneto-optical susceptibility is $\beta=1.7-2.0 \times 10^{-6} \mathrm{~m} / \mathrm{A}$. Here, we took $n=3.7$ (see Ref. 39) and the Faraday rotation $\theta_{\mathrm{F}}=0.75^{\circ}(0.013 \mathrm{rad})$.

In order to determine the magnetization compensation temperatures $T_{\mathrm{M}}$ we extracted the values of the coercive field $H_{\mathrm{c}}$ from the hysteresis loops measured as a function of temperature [see Fig. 1(b)]. The compensation temperatures are taken as those temperatures where the coercive fields diverge. As one can see from the inset in Fig. 1(b), $T_{\mathrm{M}}$ increases with an increase of $\mathrm{Gd}$ concentration, in agreement with the data from literature. ${ }^{32}$ For the samples $\mathrm{Gd}_{20} \mathrm{FeCo}$ and $\mathrm{Gd}_{28} \mathrm{FeCo}$, no compensation point was observed in the range $10<T<420 \mathrm{~K}$. The dependence of $H_{c}$ on $T$ for $\mathrm{Gd}_{20} \mathrm{FeCo}$ shows that the magnetization of the FeCo sublattice exceeds the magnetization of the Gd sublattice in the whole temperature range. In the sample $\mathrm{Gd}_{28} \mathrm{FeCo}$, the situation is opposite.

\section{METHODS}

\section{A. Experimental technique}

For studying the ultrafast magnetization dynamics during the process of all-optical reversal, we developed a single-shot time-resolved magneto-optical microscopy setup providing a 100-fs temporal resolution. This approach is based on a principle used earlier in high-speed domain wall dynamics studies. ${ }^{37,38,40}$ The setup has $1 \mu \mathrm{m}$ spatial resolution. In the experiment, a single 100-2100 fs optical pump pulse was used to excite the films (see Fig. 2). A single, less intense, 100 -fs optical probe pulse, delayed with respect to the pump pulse, was used to obtain the magneto-optical image of the sample. In this image, the areas of the sample with oppositely oriented magnetizations are seen as black and white areas due to the Faraday effect. Intermediate magnetic states are seen as different grades of gray. Repeating such a single pump (single probe measurement for various values of the delay time), we obtained images of the magnetic state of the samples at various 


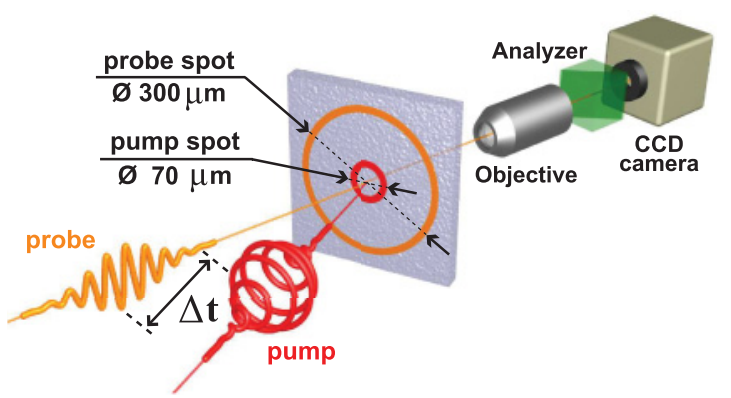

FIG. 2. (Color online) Sketch of the single-shot time-resolved magneto-optical microscopy experiment. The magneto-optical image is obtained at a CCD camera for a delay $\Delta t$ after action of a single pump pulse using a single linearly polarized probe pulse. After passing though the sample, the probe pulse passes through the microscope objective and analyzer, the transmission axis of which is perpendicular to the polarization plane of the probe pulse. Circles on the sample indicate sizes of the pump and probe spots.

moments after the arrival of the pump pulse. The same setup was also used to study the final state of the samples after the action of a single pump pulse. For this, we were either separating the pumping and probing events by a longer time delay of several seconds or were even using a continuous light source as a probe.

To obtain ultrashort laser pulses, we used a Ti:sapphire laser generating 40 -fs long pulses at a central photon energy of $E_{0}=1.54 \mathrm{eV}$ and a repetition rate of $80 \mathrm{MHz}$. After passing a regenerative amplifier a train of high peak power pulses with a repetition rate of $1 \mathrm{kHz}$ was obtained. Each pulse had a Gaussian temporal profile, full width at half maximum of $100 \mathrm{fs}$. The fluence of the pulses $(F)$ was estimated from the averaged power of the pump beam measured by a powermeter. A less intense probe beam with a central photon energy of $1.94 \mathrm{eV}$ and duration of 100 fs was generated by an optical parametric amplifier (OPA) seeded by a small fraction of the pulse from the amplifier. Note that in contrast to a similar setup described in Ref. 41, we employ a two-color pump-probe scheme, which allows a straightforward analysis of the obtained images. Using a chopper, we reduced the repetition rate of the pump and probe pulses down to $10 \mathrm{~Hz}$. Behind the chopper we placed a mechanical shutter with an opening time of $100 \mathrm{~ms}$. The combination of the chopper and the shutter, both of which were synchronized with the laser source, allowed getting a single pump and a single probe pulse. A retroreflector placed on the translational stage was used to introduce the delay between pump and probe pulses. This delay could be varied from negative values up to $3.8 \mathrm{~ns}$, with minimal steps of $10 \mathrm{fs}$. By introducing extra mirrors in the path of the probe beam, we could extend the delay time between pump and probe pulses up to $25 \mathrm{~ns}$. The pump pulse was directed at normal incidence to the sample and focused into a spot of about $50-70 \mu \mathrm{m}$ in diameter (see Fig. 2). The polarization of the pump pulses was controlled by a quarter-wave plate. Linearly polarized probe pulses are focused to a larger area of about $300 \mu \mathrm{m}$ and had an oblique angle of incidence of about $20^{\circ}$. To obtain magneto-optical images the sample was placed between two crossed polarizers. A thermoelectrically cooled CCD (charged coupled devices) camera and objectives with magnifications of 10 or 20 times, depending on the conditions of the experiment, were used to register the images. After obtaining each image, the sample was brought to the initial homogeneous magnetic state by applying a pulse of external magnetic field. Low-temperature measurements were performed using an optical cold-finger cryostat.

In addition to the time-resolved experiment, the steadystate imaging of the sample magnetization was performed at $2 \mathrm{~s}$ after an action of the laser pulse. For these purposes, the magneto-optical visualization of magnetization direction in the sample was performed using incoherent continuous white light source. Ultrashort optical pump pulses with energy of photons centered at $1.54 \mathrm{eV}$ were generated by the regenerative amplifier and used for the excitation of the sample. In contrast to the pump-probe measurements described above, in this case the duration of the pump pulses could be varied in the range of 40-2100 fs using an internal grating-based pulse compressor in the amplifier and monitored using an autocorrelator. This enabled the studies of the magnetization reversal triggered by pulses of different length.

\section{B. Theoretical methods}

The description of the dynamics of a magnetic medium during and after the excitation with a subpicosecond laser pulse comprises a complicated theoretical task. First of all, it requires an adequate model, which describes the interaction of the laser pulse with the medium. Furthermore, as the excitation with such a short laser pulse brings a medium to a transient nonequilibrium state, description of the spin dynamics of such a state goes beyond the conventional macrospin approximations in magnetism.

\section{Modeling a laser pulse impact on a medium}

As the starting point for developing a model, we assume a two-fold action of a laser pulse on the medium. First of all, we take into account the fact that a circularly polarized subpicosecond laser pulse can act on spins as an effective lightinduced magnetic field, the direction of which is determined by the helicity of the light. ${ }^{42}$ Thus one part of the impact of a laser pulse on a medium is represented by this optomagnetic field $\mathbf{H}_{\mathrm{OM}}$. Secondly, subpicosecond laser pulses are known to lead to a rapid increase of the electronic temperature followed by ultrafast demagnetization in metals. ${ }^{5}$ Therefore this part of the laser pulse impact enters our model as a short heat pulse resulting in a rapid increase of the electronic temperature $T_{\mathrm{el}}$.

To the best of our knowledge, a rigorous and sufficiently complete theoretical framework of optomagnetic effects induced in magnetic metals by femtosecond laser pulses is still under development. ${ }^{43,44}$ Therefore, here, we estimate the strength and the duration of the effective laser-induced field using existing theories and a limited number of available experimental data. In order to obtain a rough estimate for the amplitude of the effective laser-induced field, we employed a phenomenological expression for the inverse Faraday effect derived for a transparent medium in thermodynamic equilibrium: ${ }^{36,45,46}$

$$
\mathbf{H}_{\mathrm{OM}}(t, r)=\varepsilon_{0} \beta\left[\mathbf{E}(t, r) \times \mathbf{E}^{*}(t, r)\right],
$$


where $\varepsilon_{0}$ is the vacuum permittivity, $\beta$ is the magneto-optical susceptibility, and $E(t, r)$ is the envelop of the electric field of light. In isotropic media, the field $\mathbf{H}_{\mathrm{OM}}$ is directed along the wave vector of light and reverses its polarity when the helicity of the laser pulse is reversed. The amplitude of the electric field $E_{0}$ can be estimated knowing the laser pulse fluence $F$ and duration (FWHM) $\tau$ as $F / \tau \cong P_{0}=c \varepsilon_{0} / 2\left|E_{0}\right|^{2}$, where $c$ is the speed of light. In our simulations, we assumed a Gaussian distribution of the intensity of the laser beam in space. Therefore $\mathbf{H}_{\mathrm{OM}}$ was introduced as

$$
\mathbf{H}_{\mathrm{OM}}(t, r)=\sigma \frac{2 \beta F}{c \tau} f(t) e^{-\frac{r^{2}}{2 r_{0}^{2}}} \mathbf{n},
$$

where $\mathrm{c}$ is the speed of light, and $\mathbf{n}$ is the unit vector in the direction of the wave vector of light. The coefficient $\sigma$ gives the degree of circular polarization and is equal to \pm 1 and 0 for a right- $\left(\sigma^{+}\right)$, left-handed $\left(\sigma^{-}\right)$circularly polarized, and linearly polarized $(\pi)$ light, respectively. The time-dependent function $f(t)$ and the space-dependent Gaussian functions describes the temporal and the spatial profiles of the effective field and will be discussed later.

Following Eq. (1), the strength of the effective light-induced field can be calculated using the magneto-optical susceptibility $\beta$ of the medium and the amplitude of the electric field of light. We assume that in the spectral range of the laser pulse, the magneto-optical susceptibility is independent on the wavelength of light. Also, we assume that the inverse Faraday effect, described by Eq. (3) is due to interaction of light with $\mathrm{Fe}$ sublattice of GdFeCo alloy. Therefore we take the magneto-optical susceptibility to be $\beta \approx 2 \times 10^{-6} \mathrm{~m} / \mathrm{A}$ (see Sec. II). Within these approximations, circularly polarized laser pulse with fluence of $1 \mathrm{~mJ} / \mathrm{cm}^{2}$ can generate an effective magnetic field with an amplitude $H_{\mathrm{OM}} \approx 5.2 \mathrm{~T}$. For a range of intensities of $2-4.5 \mathrm{~mJ} / \mathrm{cm}^{2}$, used in the reported here experiments, the effective field has a magnitude in the range of $10.4-23 \mathrm{~T}$.

Strictly speaking, such an approach is not applicable for those cases when the intensity of light changes faster than time required for the medium to reach its thermal equilibrium. If it is not the case, the magneto-optical susceptibility $\beta$ should be considered as a time-and intensity-dependent variable. As a result, rigorous description of magneto-optical (and optomagnetic) response of a metallic medium excited by a short laser pulse is still to be developed. ${ }^{6,11,44,47,48}$ However, if the inverse Faraday effect is defined by electric-dipole transitions only, than the formulas derived for the case of thermodynamic equilibrium can still provide a reasonable approximation for an estimation of the optomagnetic effective magnetic field. Indeed, in this case, the inverse Faraday effect relies on an instantaneous response of electronic system, which reaches its quasiequilibrium faster than $100 \mathrm{fs}$ after the excitation.

The light-induced effective field is introduced in the calculations as having a Gaussian spatial profile with a radius $r_{0}$, which coincides with the spatial profile of the pump pulse. The temporal profile of this field $f(t)$ has the following form:

$$
f(t)=\left\{\begin{array}{cc}
e^{-\frac{\left(t-t_{0}\right)^{2}}{\tau^{2} / 4 \ln 2},}, & t<t_{0}, \\
e^{-\frac{\left(t-t_{0}\right)^{2}}{\left(\tau+2 \tau_{\mathrm{dca}}\right)^{2} / 4 \ln 2}}, & t>t_{0} .
\end{array}\right.
$$
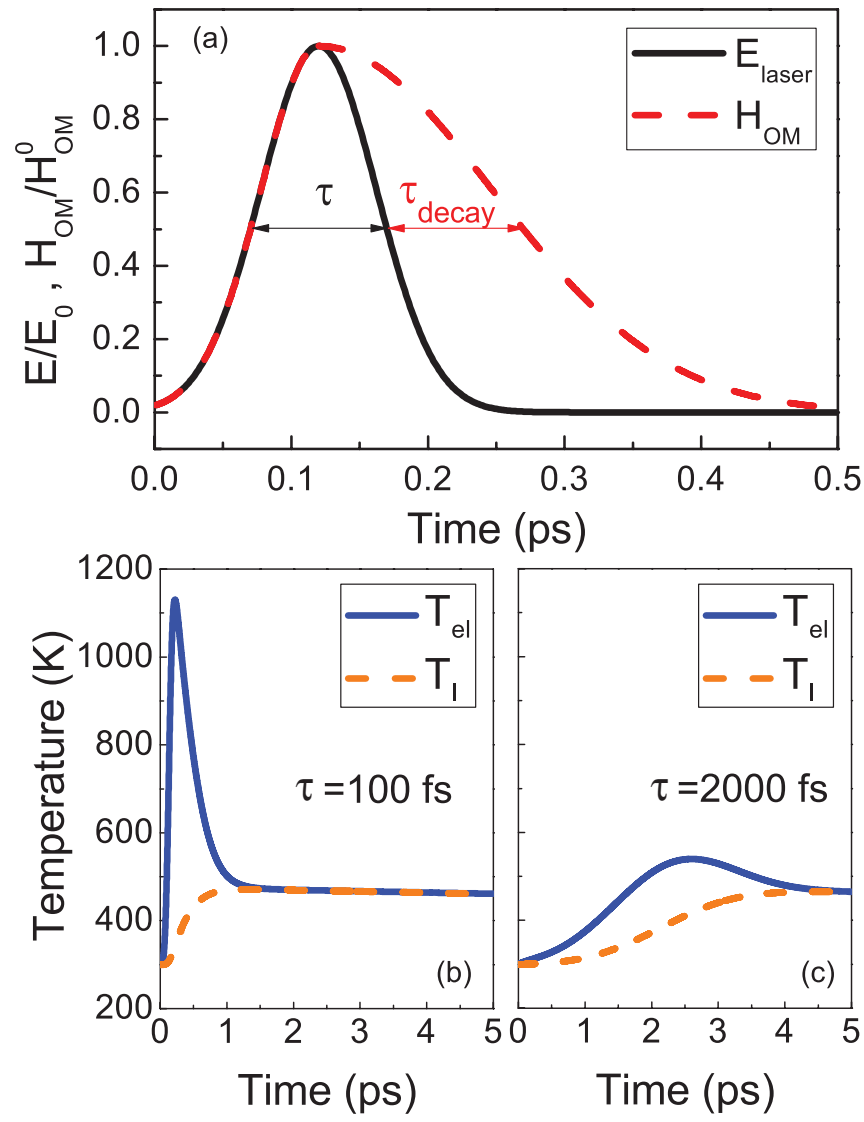

FIG. 3. (Color online) (a) The temporal profiles of the laser pulse envelope (solid line) and the optomagnetic light-induced field (dashed line). $\tau$ is the FWHM of the laser field, while $\tau_{\mathrm{OM}}=\tau+\tau_{\text {decay }}$ is the FWHM of the laser-induced effective magnetic field pulse $\mathbf{H}_{\mathrm{OM}}$. The decay time is in the range of $20<\tau_{\text {decay }}<3000 \mathrm{fs}$ (see text). (b) and (c) Time evolution of the electronic (solid line) and lattice (dashed line) temperatures $T_{\mathrm{el}}$ and $T_{\mathrm{l}}$ after an excitation with (b) 100-fs and (c) 2000-fs laser pulse as calculated from the two-temperature model (see text).

Thus, regarding the duration of this field, we assumed the time profile shown in Fig. 3. The effective light-induced magnetic field $\mathbf{H}_{\mathrm{OM}}$ builds up with no delay with respect to the laser pulse electric field. The decay of $\mathbf{H}_{\mathrm{OM}}$ is, in turn, assumed to be slower and is given by the decay time of the microscopical process responsible for the emerging of $\mathbf{H}_{\mathrm{OM}}$. At the moment, we can only estimate the limits for the decay time of $\mathbf{H}_{\mathrm{OM}}$ by considering its possible microscopical origins. The lower limit for the decay of the light-induced field is, supposedly, given by an optical coherence time, which is well below $100 \mathrm{fs}$ in metals. ${ }^{49,50}$ As one of the possible origins of the inverse Faraday effect the light-induced elliptical currents were considered. ${ }^{43,51,52}$ In this case one can expect somewhat longer decay time. So far to the best of our knowledge, no theoretical estimations for the lifetime of such currents are available. One of the possible ways to detect the presence of such currents experimentally could be studies of $\mathrm{THz}$ yield of the metallic film excited by the laser pulse. Only a few of such experiments have been preformed on magnetic metals, ${ }^{14,53}$ without, however, focusing at possible presence of the light-induced fields. Here, we use the data of these 
experiments to obtain a rough estimate for the upper limit for the decay time of the light-induced effective field. In particular, in Ref. 53, emission of polarized $\mathrm{THz}$ radiation from a thin iron film subjected to a 50-fs laser pulse excitation was measured. Since one of the possible reasons for the polarization-dependent $\mathrm{THz}$ yield is a solenoidal light-induced current, we used half a period of the lower mode of the oscillations observed in the $\mathrm{THz}$ emission as a measure of the duration of the effective light-induced magnetic field. For the case of film studied in Ref. 53, it is 3 ps. This shows that the laser-induced effective field can persist in a magnetic metal considerably longer than the duration of the laser pulse.

The second contribution to the laser pulse impact on the medium is introduced as a heat pulse, the duration of which is defined by the laser pulse duration. As in the case of the optomagnetic pulse, the spatial profile of the heat pulse is Gaussian and coincides with the profile of the laser pulse,

$$
P(t, r)=P_{0} e^{-\frac{r^{2}}{2 r_{0}^{2}}} e^{-\frac{\left(t-t_{0}\right)^{2}}{\tau^{2} / 4 \ln 2}} .
$$

The action of this pulse results in a rapid increase of the electron temperature $T_{\mathrm{el}}$ of the system. To calculate this response, we used the two-temperature model, ${ }^{54}$

$$
\begin{gathered}
C_{\mathrm{el}}\left(T_{\mathrm{el}}\right) \frac{d T_{\mathrm{el}}}{d t}=-G_{\mathrm{el}-\mathrm{ph}}\left(T_{\mathrm{el}}-T_{1}\right)+P(t, r), \\
C_{\mathrm{l}}\left(T_{1}\right) \frac{d T_{1}}{d t}=-G_{\mathrm{el}-\mathrm{ph}}\left(T_{1}-T_{\mathrm{el}}\right)-C_{1} \frac{T_{1}-T_{0}}{\tau_{\mathrm{th}}},
\end{gathered}
$$

where $T_{\mathrm{el}}$ and $T_{1}$ are the temperatures of the electronic and lattice reservoirs, $C_{\mathrm{el}}$ and $C_{1}$ are the electronic and lattice specific heats, respectively, and $G_{\mathrm{el}-\mathrm{ph}}$ is the electron-phonon coupling constant. The time constant $\tau_{\text {th }}=50 \mathrm{ps}$ describes the relaxation back to the initial temperature $T_{0}$. The parameters used in the model were assumed to be typical for a transition metal. ${ }^{55}$ In this model, after the action of a laser pulse, the electron temperature $T_{\mathrm{el}}$ increases from the initial temperature (equilibrium temperature of the sample) up to $T_{\mathrm{el}}^{*}$ within the duration of the laser pulse. The value of $T_{\mathrm{el}}^{*}$ is defined by the fluence of the laser pulse and by the electron heat capacity $C_{\mathrm{el}}$, which is $C_{\mathrm{el}}=\gamma T_{\mathrm{el}}$ with $\gamma=700 \mathrm{~J} / \mathrm{m}^{3} \mathrm{~K}^{2}$. The increase is followed by a decrease of $T_{\mathrm{el}}$ due to the electron-phonon relaxation. The characteristic time of this relaxation is determined by the electron-phonon coupling constant $G_{\text {el-ph }}$, which is assumed to be $1.7 \times 10^{18} \mathrm{~J} / \mathrm{Ks}$. In Figs. 3(b) and 3(c), we show the calculated dynamics of the $T_{\mathrm{el}}$ and $T_{1}$ for heat pulses of durations 100 and $2000 \mathrm{fs}$.

\section{Modeling ultrafast nonequilibrium spin dynamics}

Atomistic simulations may allow one to calculate the response of the spin system to the combined action of the ultrafast heating and the optomagnetic field $\mathbf{H}_{\mathrm{OM}}$. In this approach, the spin system interacts with a heat bath. Therefore it is the change of the heat-bath temperature which triggers the ultrafast demagnetization. In the model, the demagnetization occurs due to angular momentum transfer from the spin system to the heat bath. Despite intensive experimental and theoretical research on this matter the microscopical origin of the ultrafast laser-induced demagnetization has not been clarified yet. Therefore it is questionable if the heat-bath can be associated with a free-electron gas or should be assigned to another reservoir of energy and angular momentum. Recently, it was demonstrated that such a model, where the heat bath was associated with a free-electron gas, allows to describe well the experimentally observed ultrafast magnetization dynamics in FePt alloys. ${ }^{19}$ The potential of this approach to explain and even predict ultrafast spin dynamics was demonstrated for the case of GdFeCo as well. ${ }^{29}$ Furthermore, such atomistic simulations confirmed the results of element-specific studies of ultrafast laser-induced magnetization dynamics in the alloys showing that $\mathrm{Gd}$ and $\mathrm{Fe}$ demagnetize on significantly different time scales. Consequently, in GdFeCo alloys the Gd sublattice can play the role of a reservoir of angular momentum during the process of demagnetization of the Fe sublattice and vice versa. In this case, the angular momentum effectively stays in the spin system.

The dynamics of each spin is described by the stochastic Landau-Lifshitz-Gilbert (LLG) equation, where the total effective field entering the LLG equation contains contributions from a Heisenberg exchange, a crystalline anisotropy, and the optomagnetic field $\mathbf{H}_{\mathrm{OM}}$. This atomistic approach, however, does not allow to calculate the behavior of a large ensembles of spins. Nevertheless, it allows to calculate the equilibrium magnetization, the perpendicular and parallel magnetic susceptibilities as well as the exchange stiffness of the spin system, required for the description of the magnetization dynamics in the case, when neither the length nor even the equilibrium value of the magnetization are conserved. ${ }^{19}$

In our multiscale model, the behavior of the averaged magnetization $\mathbf{m}$ after the action of the laser pulse is described using the Landau-Lifshitz-Bloch (LLB) equation. ${ }^{19,56}$ The temperature dependence of the uniaxial anisotropy constant $K_{\mathrm{u}}$ is introduced via a temperature-dependent transverse susceptibility. ${ }^{19}$ The room-temperature value of $K_{u}$ is taken to be $6.05 \times 10^{5} \mathrm{~J} / \mathrm{m}^{3}$ and the exchange stiffness is chosen such that it leads to the Curie temperature of $500 \mathrm{~K}$. The microscopic Gilbert damping constant is assumed to be 0.1 . Solely ferromagnetic behavior is considered here so that the details of the ferrimagnetic spin structure cannot be simulated. All methods used are described in details in Ref. 19.

Using this micromagnetic approach, we are able to perform calculations of the spin dynamics of extended systems accounting for spatially inhomogeneous excitation process on a micrometer length scale. This approach takes long-range dipolar interactions into account, which are calculated with the aid of the well established fast-Fourier transformation. The parallelized code used in the calculations allowed to simulate systems with up to $10^{7}$ macro-spins (cells). The volume of the modeled spin system is up to $10 \mu \mathrm{m} \times 10 \mu \mathrm{m} \times 5 \mathrm{~nm}$, which is comparable to the size of the systems, studied in the experiments. For these micromagnetic simulations, the heat pulse and the pulse of the optomagnetic field were modeled by a Gaussian spatial profile as explained above. In addition to this computation-time-demanding large-scale simulations, we also perform single-macrospin simulations, where the macrospin represents a single-domain volume of $\left(30 \mathrm{~nm}^{3}\right)$. Note, that at this stage, we neglect the spatial distribution of the $\mathbf{H}_{\mathrm{OM}}$ in Eq. (2) and of $T_{\mathrm{el}}$. 


\section{RESULTS AND DISCUSSION}

\section{A. Determination of the laser pulse fluence for the helicity dependent reversal}

\section{Experiment}

To investigate how the process of all-optical magnetization reversal depends on the laser pulse fluence, we studied the final state of the magnetization after the action of a single laser pulse as a function of the laser pulse fluence. Figure 4(a) shows the steady-state magneto-optical images of the $\mathrm{Gd}_{26}$ FeCo sample obtained after the sample was subjected to single circularly polarized pulses of various fluences.

As one can see from Fig. 4(a), the change of the laser pulse fluence $(F)$ leads to a change of the magnetic state observed after the action of the pulse. If $F$ is below $\sim 3.14 \mathrm{~mJ} / \mathrm{cm}^{2}$, no reversal is observed (not shown). If $F$ is about $3.14 \mathrm{~mJ} / \mathrm{cm}^{2}$, all-optical helicity-dependent reversal is observed. As $F$ is increased by a relatively small value, the reversal of magnetization is observed after the action of laser pulses of both helicity, and even a linearly polarized pulse can trigger the reversal. However, the size of the spot appears to be slightly larger, if the helicity of the laser pulse favors the helicity-dependent reversal at lower fluence. This is demonstrated in the last row in Fig. 4(a). At laser fluences above $\sim 4.7 \mathrm{~mJ} / \mathrm{cm}^{2}$ the formation of a multidomain state is observed. We note in Fig. 4(b) that in the Gaussian spatial profile of the laser pulses of high fluence $\left(F>4.5 \mathrm{~mJ} / \mathrm{cm}^{2}\right)$ we can distinguish four regions, corresponding to the four types of the impact of a laser pulse on the magnetic medium: no reversal, helicity-dependent all-optical reversal, helicity-independent reversal, and the formation of a multidomain state. It is important to stress, that the size of the area where the helicity-dependent magnetization reversal occurs is much smaller than the size of the laser spot itself.

An analogous dependence of the reversal on the laser pulse fluence was observed in several other samples, with absolute values of the fluence being slightly different, as discussed in Sec. IV D. To examine the range, where the helicity-dependent reversal is observed, we studied the laser pulse fluence dependence of the probability for the magnetization to be reversed after the action of a single right- $\left[\mathcal{P}\left(\sigma^{+}\right)\right]$or lefthanded $\left[\mathcal{P}\left(\sigma^{-}\right)\right]$circularly polarized pulse. In more details, for each initial magnetization direction, laser pulse polarization and fluence $N$ attempts to switch the magnetization by a single laser pulse were taken. Before each attempt, the sample was brought to the initial monodomain state by applying a pulse of magnetic field. If the switching was observed in $M$ cases, then the probability of reversal for this particular set of conditions was taken as $(100 \mathrm{M} / N) \%$. The result for the sample $\mathrm{Gd}_{22} \mathrm{FeCo}$ is shown in Fig. 4(c), where these probabilities and the all-optical switchability, i.e., $\mathcal{P}\left(\sigma^{+}\right)-\mathcal{P}\left(\sigma^{-}\right)$, are plotted as a function of the laser pulse fluence. As one can see, there is a narrow range of laser pulse fluences $(\sim 10 \%)$, where the switchability is nonzero $\mathcal{P}\left(\sigma^{+}\right)-\mathcal{P}\left(\sigma^{-}\right)>0$, i.e., the probability for a helicity-dependent reversal to occur is finite. In the middle of this range, the switchability reaches $100 \%$. The corresponding FWHM of this range is of $6 \%$. At these conditions, the direction of the magnetization in the final state is unambiguously determined by the helicity of the

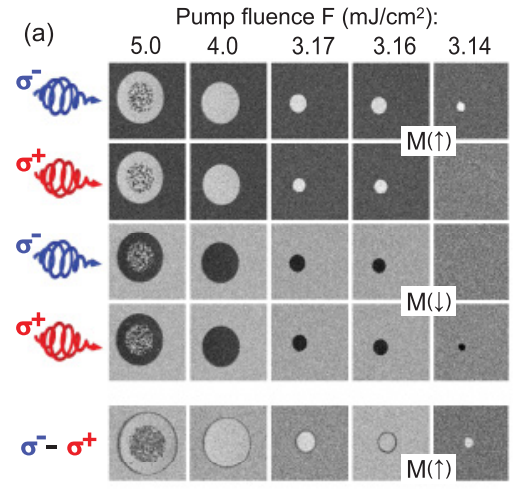

(b)
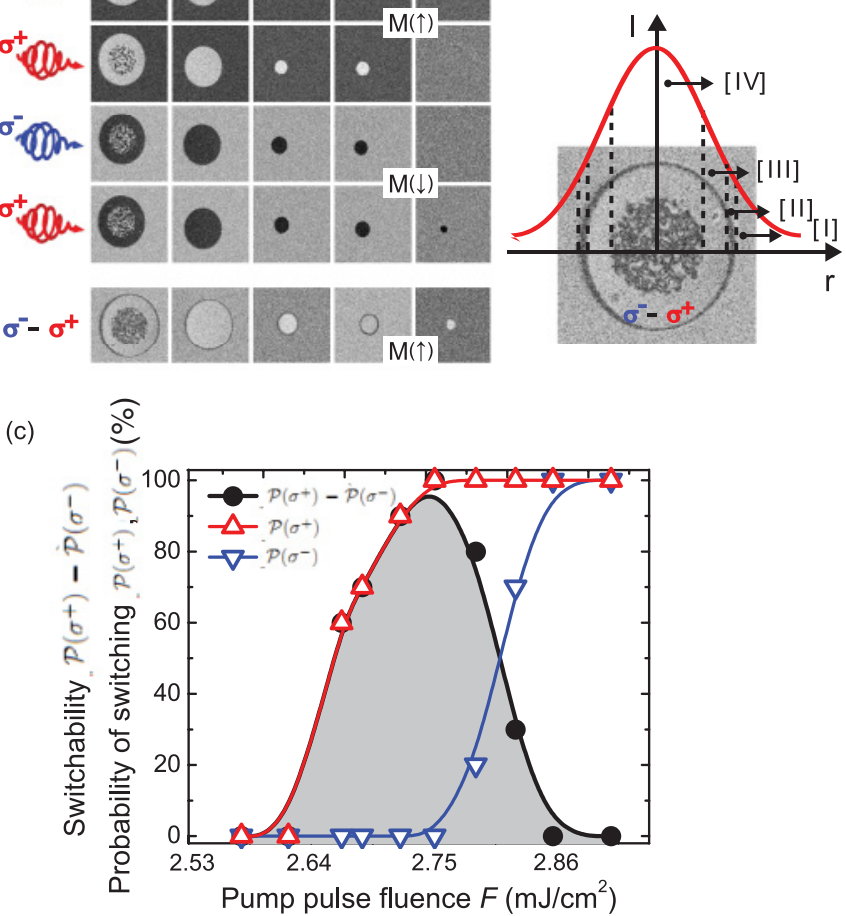

FIG. 4. (Color online) (a) Rows 1-4: steady-state magnetooptical images of the sample $\mathrm{Gd}_{26} \mathrm{FeCo}$ at room temperature obtained after the action of single $100 \mathrm{fs} \sigma^{+}$- and $\sigma^{-}$-polarized laser pulses with fluence $F$. Before each excitation event, the sample was brought to a single-domain initial state by applying a magnetic field pulse of a proper direction. The initial direction of magnetization was "up" for images in rows 1 and 2, and "down" in rows 3 and 4. Each of the images is the result of the substraction of the image obtained before the excitation with the single laser pulse from the image obtained at $\sim 2 \mathrm{~s}$ after the excitation. Images were obtained using a white light source. The last row shows the substraction of the images in rows 1 and 2 obtained with pulses of opposite helicities for each specific fluence. (b) Schematic presentation of the laser pulse fluence distribution and the reversal for $F=5 \mathrm{~mJ} / \mathrm{cm}^{2}$ : [I] fluence range where no all-optical reversal is observed, [II] helicity-dependent reversal, and [III] helicity-independent reversal and [IV] multidomain state. (c) Probabilities that the magnetization is reversed after the action of a single left- $\left[\mathcal{P}\left(\sigma^{-}\right)\right]$and right-handed $\left[P\left(\sigma^{+}\right)\right]$circularly polarized laser pulse (open triangles) and all-optical switchability ${ }^{26}$ $\mathcal{P}\left(\sigma^{+}\right)-\mathcal{P}\left(\sigma^{-}\right)$(closed circles) vs $F$ in the sample $\mathrm{Gd}_{22} \mathrm{FeCo}$. For obtaining the shown probabilities, the statistics over ten switching events was used. Increasing the number of events up to 100 was affecting neither the FWHM nor the maximum switchability.

laser pulse. When the laser pulse fluence is above the reversal window, the reversal into a single domain state still occurs, but appears to be independent of the helicity of the laser pulse.

Thus, from the study of the impact of laser pulses of various fluences and helicities on magnetization of the GdFeCo alloy, we can conclude that the all-optical reversal occurs only when the medium is subjected to a circularly polarized pulse with its fluence being within a narrow range, or reversal window. Below this window, no reversal occurs. Above this window the reversal is insensitive to the polarization of the 
laser pulse, which is a manifestation of the fact that the information about the light polarization is lost. In the following sections, we focus our paper on all-optical helicity-dependent magnetization reversal only. We add that the narrowness of the reversal window is consistent with the small size of the domain where the magnetization is reversed all-optically [see Fig. 4(b)]. Due to the Gaussian spatial profile of the laser spot, only in the very center of the spot the laser fluence falls within the reversal window.

\section{Modeling}

In order to understand how the observed helicity-dependent magnetization reversal can occur, we performed multiscale calculations of the dynamics of the ferromagnetically coupled spins subjected to the combined action of the heat and optomagnetic pulse, as described in Sec. III.

In the two-temperature model, the action of the 100-fs laser pulse with an energy density $W$ leads to a rapid increase of the electron temperature $T_{\mathrm{el}}$ from $300 \mathrm{~K}$ up to $T_{\mathrm{el}}^{*}$. This increase is followed by a relaxation of $T_{\mathrm{el}}$ due to the electron-phonon interactions with a characteristic time of $0.5 \mathrm{ps}$. The quasiequilibrium electronic temperature achieved after this relaxation is determined by the laser energy density $W$ and, consequently, by the peak electronic temperature $T_{\mathrm{el}}^{*}$, as shown in Fig. 5(a). Figure 5(b) shows the calculated dynamics using the LLB equation of a single macro spin, representing the averaged magnetization of a $(30 \mathrm{~nm})^{3}$ spin system for three different energy densities $W$ and optomagnetic field of an amplitude $H_{\mathrm{OM}}$ for a duration of $\tau_{\mathrm{OM}}=250 \mathrm{fs}$. This field is directed antiparallel with respect to the initial orientation of the magnetization. As one can see, for the particular value of $W=0.57 \mathrm{GJ} / \mathrm{m}^{3}\left(T_{\mathrm{el}}^{*}=1129 \mathrm{~K}\right)$ the magnetization reverses. The reversal occurs via a linear path and does not involve precession, i.e., the transverse components of the magnetization remain zero (not shown). Instead, at the very early stage of the reversal process the magnetization is nearly quenched. It is important to stress, that atomistic-simulationsbased examination of the magnetic state achieved as a result of the ultrashort laser pulse, ${ }^{57}$ revealed that this state is principally different from the paramagnetic equilibrium state. In fact, the laser induced demagnetization results in a magnetic state with no net magnetization, i.e., the state is still characterized by a correlation length, which, however, is of order of a few nanometers or less. As it was shown previously, ${ }^{27}$ for the linear dynamics of the spin system, achieving a nearly quenched magnetization state is crucial. In this state the dynamics of the spins is governed by the longitudinal damping. This favors the reversal of magnetization by the short strong pulse of the optomagnetic field $\mathbf{H}_{\mathrm{OM}}$ directed antiparallel with respect to M. We note that this is in contrast to the precessional switching of the magnetization, which is governed by the transverse damping.

As it can be seen from Fig. 5(b), the final state of the magnetization is mainly defined by $T_{\mathrm{el}}^{*}$, and, consequently, by energy density $W$. For the low peak electron temperature (e.g., $T_{\mathrm{el}}^{*}=970 \mathrm{~K}$ ), the spin system reaches a state with nearly quenched magnetization, but later on relaxes back to the initial state, i.e., magnetization reversal does not occur. Thus, for the given amplitude and duration of $\mathbf{H}_{\mathrm{OM}}$, the temperature $T_{\mathrm{el}}$

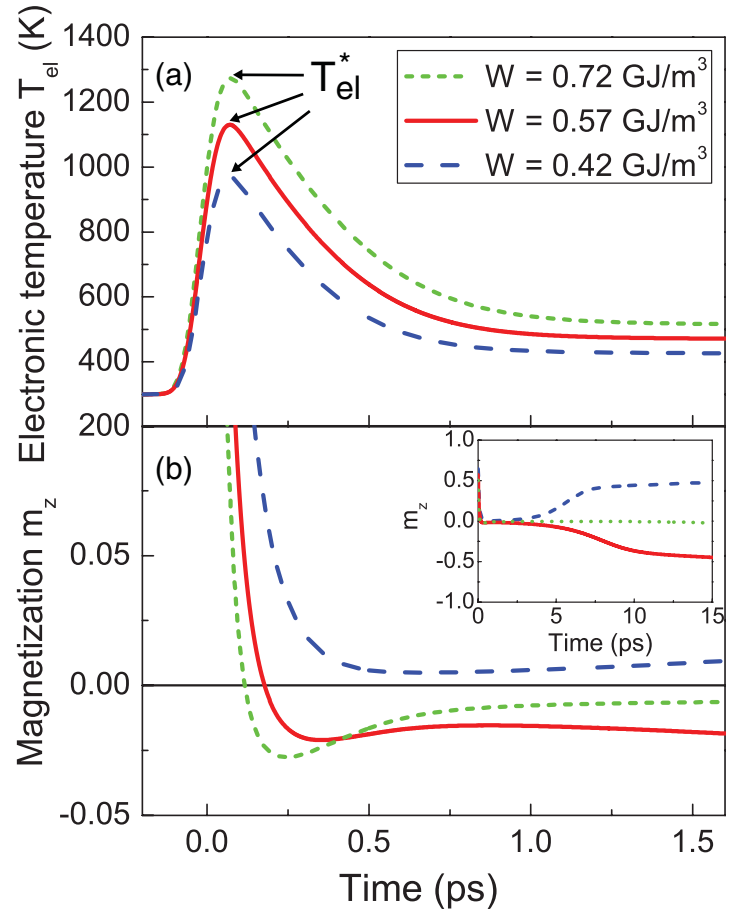

FIG. 5. (Color online) (a) Electronic temperature as a function of time, as calculated from the two-temperature model for the laser pulse energy densities $W=0.42$ (blue dashed line), 0.57 (red solid line), and $0.72 \mathrm{GJ} / \mathrm{m}^{3}$ (green short-dashed line). The corresponding peak electronic temperatures are $T_{\mathrm{el}}^{*}=970,1129$, and $1273 \mathrm{~K}$, respectively. (b) Dynamics of the longitudinal component of the magnetization of the $(30 \mathrm{~nm})^{3}$ spin system for these three different laser pulse energy densities and corresponding optomagnetic field amplitudes $\left(H_{\mathrm{OM}}=18.8 \mathrm{~T}\right.$ for the lowest fluence) with duration $\tau_{\mathrm{OM}}=250 \mathrm{fs}$. The inset shows the dynamics for a time scale up to $15 \mathrm{ps.}^{26}$

achieved within the duration of the effective field pulse is not sufficient for the occurrence of the linear reversal. The spin system relaxes into the state with reversed magnetization only for certain intermediate values of $T_{\mathrm{el}}^{*}$. If $T_{\mathrm{el}}^{*}$ is too high, the calculations give a final state with zero magnetization. This shows that the highly nonequilibrium state, with $T_{\mathrm{el}}$ sufficient for the linear reversal, should not persist for too long after the end of the effective magnetic field pulse. Otherwise the effect of the field pulse action, namely, the nucleation of the phase with the reversed magnetization, will be lost. In the latter case, the system is expected to relax back to a state with random orientation of magnetization, yielding a multidomain state.

The width of the laser pulse energy density range, or the reversal window, where the magnetization reversal via the linear mechanism can occur, is strongly influenced by changes of the parameters of the optomagnetic field pulse $\mathbf{H}_{\mathrm{OM}}$. In Fig. 6(a), we examine the state of our nanoelement $10 \mathrm{ps}$ after the combined action of the heat and optomagnetic pulses for different laser energy densities and optomagnetic field pulse durations. The heat pulse duration was fixed at $100 \mathrm{fs}$. As can be seen from Fig. 6(a), there is a region in that parameter space where the reversal occurs. As the duration of the effective field pulse increases, the reversal occurs in a broader window of laser fluences. The lower limit of $\tau_{\mathrm{OM}}$, at which the 


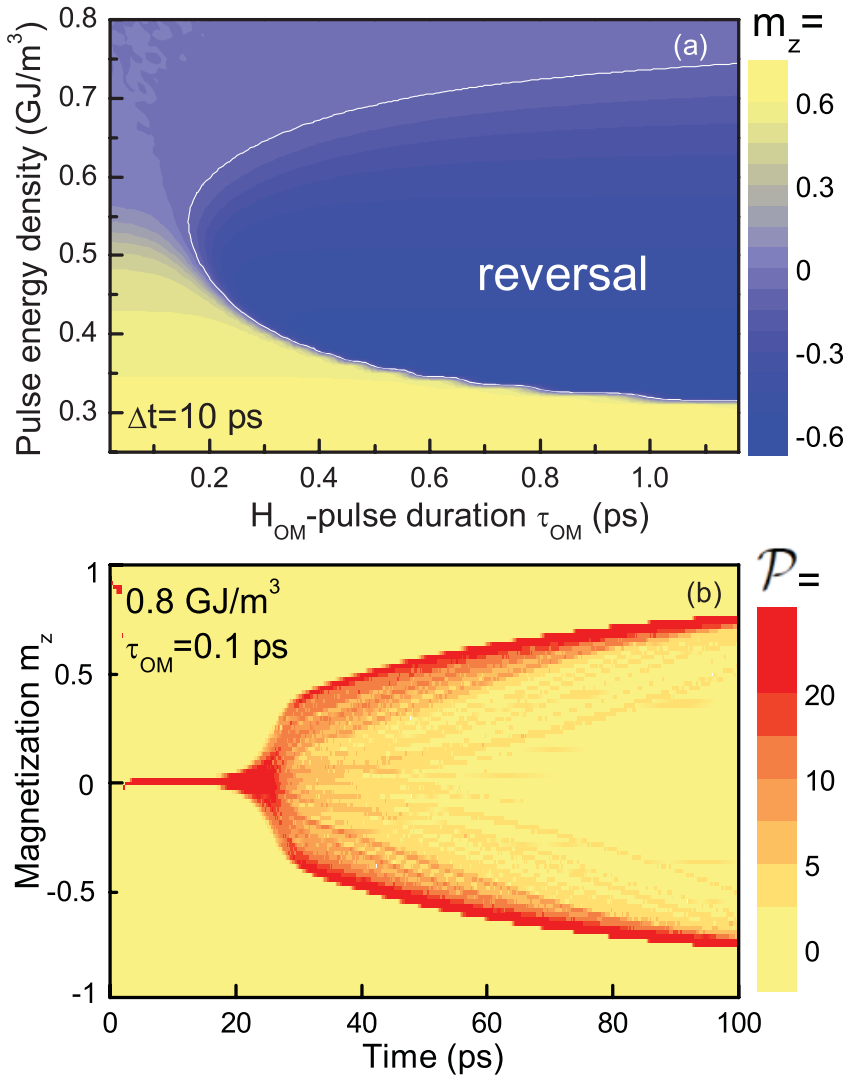

FIG. 6. (Color online) (a) Phase diagram showing the $z$ component of the averaged magnetization $m_{\mathrm{z}}$ of the $(30 \mathrm{~nm})^{3}$ volume $10 \mathrm{ps}$ after the action of the laser pulse with given energy density and optomagnetic pulse duration. ${ }^{26}$ The magnitude of the optomagnetic field scales with laser pulse energy as $H_{\mathrm{OM}}=12-32 \mathrm{~T}$ for $W=$ $0.3-0.8 \mathrm{GJ} / \mathrm{cm}^{3}$. The laser pulse duration $\tau$ is considered to be $100 \mathrm{fs}$. The color code for the $m_{\mathrm{z}}$ values is given on the right-hand side of the figure. The initial value of $m_{\mathrm{z}}$ is +1 . The solid line corresponds to $m_{\mathrm{z}}=0$ and separates the region with finite probability for the final state to be reversed. (b) Statistical analysis of the time evolution of the $m_{z}$ component of the magnetization at high pulse energy density $\left(W=0.8 \mathrm{GJ} / \mathrm{m}^{3}\right)$. The color code for the probabilities for $m_{z}$ to have a certain value at a certain time delay is shown on the right-hand side of the figure (in \%). The resulting average value of $m_{z}$ at such a laser pulse fluence is zero at any time delay, as shown in (a) and Fig. 5(b).

reversal can be realized, is strongly influenced by the material constants entering the calculations (see Sec. III). In particular, an increase of the electron-phonon coupling constant $G_{\text {el-ph }}$, governing the dynamics of electronic and lattice response to the heat pulse, leads to a reduction of the minimum field pulse duration. For the parameters used in the calculations, the minimal duration of the optomagnetic field, for which the reversal occurs, is $250 \mathrm{fs}$. Therefore despite the fact that the parameters of the laser-induced effective field $\mathbf{H}_{\mathrm{OM}}$ are not fully defined at this stage, the results in Fig. 6(a) show that the regime of linear reversal is achievable for a rather wide range of optomagnetic field parameters. We note that the effective field duration of 250 fs is well below the roughly estimated upper limit of 3 ps for the effective laser induced field (see Sec. III).
For any optomagnetic pulse durations $\tau_{\mathrm{OM}}$, there is a laser pulse energy, above which the calculated final state for the magnetization is zero [see Figs. 5(b) and 6(a)]. Detailed analysis of the dynamics of the $(30 \mathrm{~nm})^{3}$ spin ensemble at $W=0.8 \mathrm{GJ} / \mathrm{m}^{3}$ shows that there are equal probabilities for the magnetization to reemerge in both $+z$ and $-z$ directions after an excitation with such a pulse. This is illustrated in Fig. 6(b), where the statistics of the time evolutions of the $m_{\mathrm{z}}$ component is shown. It is seen that at high laser pump fluences the final state of the magnetization is defined by neither optomagnetic field pulse nor by the initial magnetic state. The latter fact corresponds to the loss of the magnetic memory studied in Ref. 57.

To summarize, the model shows that the action of laser pulses with a duration above a certain limit results in magnetization reversal. In a good agreement with our experimental results, this process can be realized only in a narrow range of the laser pulse fluences. Most importantly, our model shows that the magnetization during the reversal process follows a linear trajectory passing through a state with no net magnetization. The latter can be seen as a distinct feature of the laser-induced reversal process. The model also provides quite intriguing predictions about the dynamics of the all-optical magnetization reversal, showing that it may occur within just a few picoseconds. As it can be seen from Fig. 5(b), under the conditions favoring linear reversal $\left(T_{\mathrm{el}}^{*}=1129 \mathrm{~K}\right)$ almost $50 \%$ of the state with reversed magnetization is reconstructed already after $\sim 10 \mathrm{ps}$. In order to verify whether the all-optical reversal indeed occurs via a demagnetized state and whether its dynamics is indeed that fast, time-resolved experimental studies should be performed.

\section{B. Dynamics of the all-optical helicity-dependent reversal}

\section{Experiment}

In order to identify the path which the magnetization follows after excitation with a circularly polarized pulse with the fluence [see Fig. 4(c)], we employed the single-shot time resolved microscopy technique, described in Sec. III A. Figure 7(a) shows the single-shot magneto-optical images of the $\mathrm{Gd}_{24} \mathrm{FeCo}$ sample obtained at room temperature at various time delays between pump and probe pulses. Experiments have been performed for various combinations of the pump pulse polarization and two initial orientations of the magnetization. It is seen that within the first picosecond, pump pulses of both helicities bring the originally magnetically ordered medium into a strongly nonequilibrium state with no measurable net magnetization, seen as a gray area [see the second column of Fig. 7(a)]. In the following few tens of picoseconds, either the medium relaxes back to the initial state or a small $(\sim 5 \mu \mathrm{m})$ domain with a reversed magnetization is formed. We especially note that we see no sign of precession during the whole reversal process. If a precession of the magnetization would take place, the appearance of an in-plane component of magnetization, exceeding $\sim 10 \%$ of the equilibrium magnetization, would become visible in the time-resolved images due to the oblique incidence of the probe beam (see Sec. III). Appearances of smaller in-plane components cannot be resolved in these experiments due to limited sensitivity. Therefore, in order to confirm the absence of precession, we performed conventional 


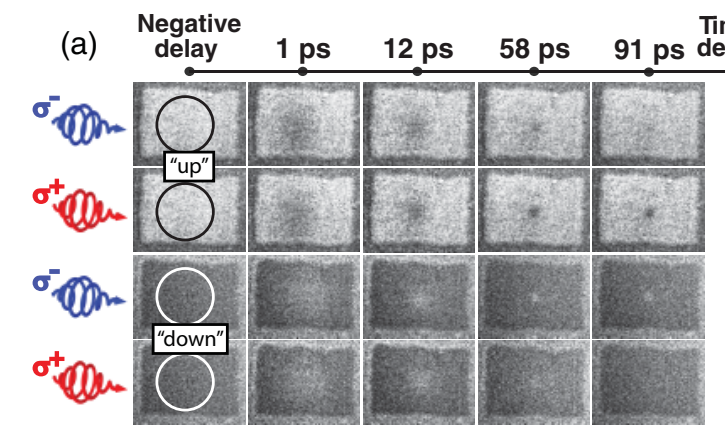

(c)

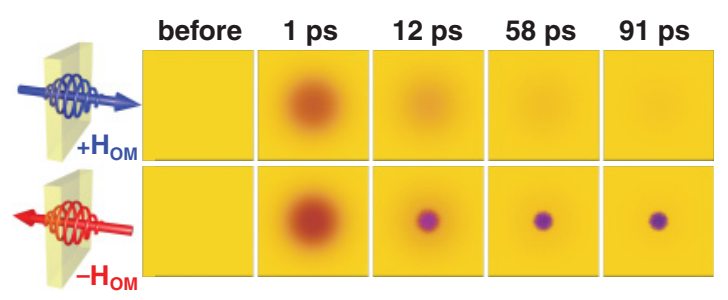

Time

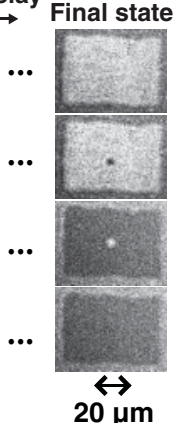

$20 \mu \mathrm{m}$

(b)
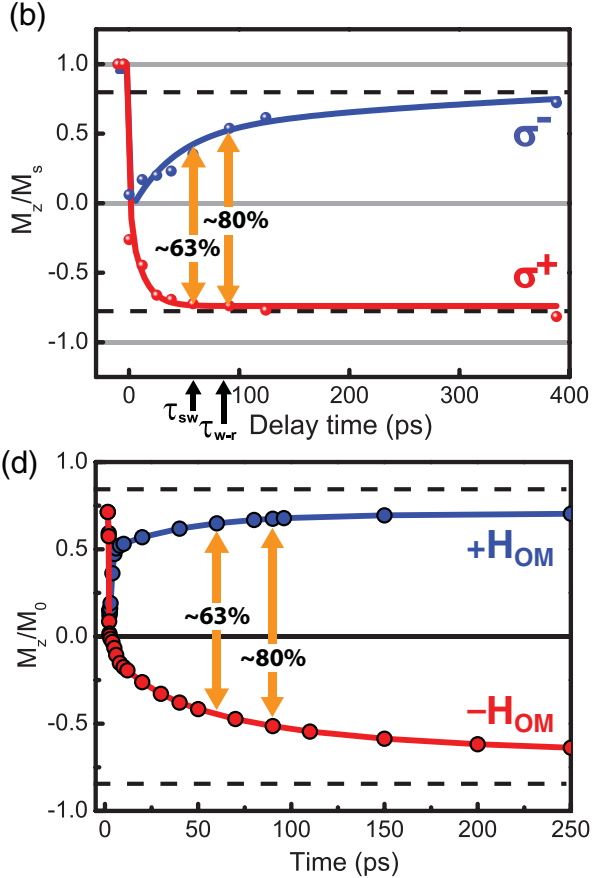

FIG. 7. (Color online) (a) The magnetization evolution in $\mathrm{Gd}_{24} \mathrm{Fe}_{66.5} \mathrm{Co}_{9.5}$ after the excitation with $\sigma^{+}$and $\sigma^{-}$circularly polarized pulses at room temperature. ${ }^{26}$ The domain is initially magnetized up (white domain) and down (black domain). The last column shows the final state of the domains after a few seconds. The circles show areas, where the effect of the laser pulse on the magnetic state is detected within the sensitivity of the setup. Note that the pump spot size is of 50-70 $\mu \mathrm{m}$ and larger than the images. (b) The averaged magnetization in the switched areas $(\sim 5 \mu \mathrm{m})$ after $\sigma^{+}$and $\sigma^{-}$laser pulses, as extracted from the single-shot images in Fig. 7(a) for the initial magnetization up. ${ }^{26}$ (c) Distributions of the $z$ component of the magnetization across the $10 \mu \mathrm{m} \times 10 \mu \mathrm{m} \times 5 \mathrm{~nm}$ ferromagnetic film at different time delays after the combined action of a $100 \mathrm{fs}$ long laser pulse and a $250 \mathrm{fs}$ long optomagnetic field pulse as obtained from the multimacrospin simulations. The peak electronic temperature is $T_{\mathrm{el}}^{*}=1129 \mathrm{~K}$. Yellow, red, and blue (in the black and white version: light gray, gray, and dark gray) regions correspond to positive, zero, and negative values of $m_{\mathrm{z}}$, respectively. (d) The averaged magnetization in the switched areas ( $\left.\sim 0.5 \mu \mathrm{m}\right) \mathrm{vs}$ time.

(non-single-shot) magneto-optical pump-probe experiments, where the pulsed magnetic field was used to reset the initial state of magnetization after each pump-probe event. In these experiments, the sensitivity is increased compared to the single-shot imaging, owing to the averaging over several hundred pulses and lock-in detection technique. Again, very fast decrease of the magnetization was observed without any sign of precession. Therefore the gray areas seen at short delay times correspond to the quenching of both out-of-plane and in-plane components of magnetization. It appears that the all-optical helicity-dependent reversal proceeds via a state with nearly quenched magnetization. This observation fully agrees with our model of linear reversal.

From the results shown in Fig. 7(a), it follows that the reversal process is inhomogeneous across the area subjected to the laser pulse. In order to characterize the observed dynamics quantitatively and to estimate the actual switching time, we calculated the averaged value of the magnetization at the various delays in the area of $\sim 5 \mu \mathrm{m}$. This area corresponds to the domain with the reversed magnetization in the final state [see right column in Fig. 7(a)]. The result is shown in Fig. 7(b). Already at delays shorter than 100 ps, a clear magnetic contrast is observed between the states reached after excitations with laser pulses of different helicities. This contrast is slightly different from the contrast between the final states [the last column in Fig. 7(a)], as clearly seen from Fig. 7(b). This can be ascribed to the laser-induced heating of the sample followed by a slow ( $>1 \mathrm{~ns}$ ) heat diffusion. To take into account the heat-induced change of the magnetization in the metastable magnetization states, we introduce two asymptotic levels [see dashed lines in Fig. 7(b)]. The characteristic time of switching $\tau_{\text {sw }}$ can be identified as the time required to reconstruct $63 \%$ $\left(1-e^{-1}\right)$ of the difference between the two metastable states. After $1.5 \tau_{\mathrm{sw}}$, the difference reaches $80 \%$. Figure 7(a) shows that this time can indeed be reliably assumed as the period required for a write-read event. Thus, we can conclude that the switching time and the write-read time for the case shown in the Figs. $7(\mathrm{a})$ and $7(\mathrm{~b})$ are $\tau_{\mathrm{sw}}=60 \mathrm{ps}$ and $\tau_{\mathrm{w}-\mathrm{r}}=90 \mathrm{ps}$, respectively.

\section{Modeling}

Experimental results shown in Figs. 7(a) and 7(b) clearly demonstrate that the all-optical helicity-dependent reversal indeed possesses the main features expected for the linear reversal as described in the previous Sec. IV A. However, one can notice a clear discrepancy between reversal times observed numerically [see Fig. 6(a)] and experimentally [see Fig. 7(b)]. These calculations were done for a small magnetic element, neglecting spatial inhomogeneities of both optomagnetic and 
heating pulses. In order to investigate the linear reversal in a magnetic film of micrometer size under realistic excitation conditions, we performed multimacrospin LLB calculations (see Sec. III). We considered a $10 \mu \mathrm{m} \times 10 \mu \mathrm{m} \times 5 \mathrm{~nm}$ ferromagnetic film subjected to a heat pulse and optomagnetic field pulse [see Eq. (2)] of Gaussian spatial profile with $r_{0}=2.1 \mu \mathrm{m}$. The laser fluence and duration as well as the optomagnetic field duration are chosen to favor linear reversal (see Fig. 5). The calculated distributions of the magnetization at various time delays for two opposite $\mathbf{H}_{\mathrm{OM}}$ are shown in Fig. 7(c). Figure 7(d) shows the calculated time evolution of the $z$ component of the magnetization in the center of the magnetic element shown in Fig. 7(c). Comparison of the calculated and experimental results shows that the spatial profile of the process of the relaxing magnetic state is determined by the spatial distribution of the laser pulse fluence, which defines the distribution of both the electronic temperature and the optomagnetic field. The switching times obtained for the larger element appear to be close to those obtained in the experiment [see Figs. 7(b) and 7(d)].

Thus, using both experimental results and multiscale modeling, we confirm that the all-optical helicity-dependent reversal can be a result of ultrafast laser-induced heating accompanied by the action of the effective laser-induced magnetic field. The latter is responsible for the dependence of the reversal on the laser pulse helicity. In this process of the all-optical reversal, the magnetization follows an unusual linear path, not involving precessional motion but passing through the state with no net magnetization. Simulations show that the nucleation of the phase with the reversed magnetization happens on an ultrafast time scale of just $\sim 1 \mathrm{ps,} \mathrm{which}$ is followed by a slower relaxation to the equilibrium state. Even though the write-read time achieved in the experiment appears to be comparable to the fastest switching in an external magnetic field or by a spin-polarized current, our model shows that the write-read time can be even faster and reach values as low as $\sim 10$ ps.

The consideration above, however, leaves two important questions open. First, as the realistic parameters, strength and duration, of the laser-induced effective field $\mathbf{H}_{\mathrm{OM}}$ are still under discussion, it is important to investigate what would happen to the helicity-dependent all-optical reversal when these parameters are changed. Second, so far, neither in the experiments nor in the modeling, the ferrimagnetic structure of the GdFeCo alloys was taken into account. Consequently, it is interesting to verify if the ferrimagnetism of $\mathrm{GdFeCo}$ plays a role in the reversal process. In particular, whether the write-read times close to those predicted by the model can be achieved by tailoring the properties of the medium.

\section{Helicity-dependent all-optical reversal as a function of the laser pulse duration}

Parameters of a laser pulse affect both the demagnetization process and the optomagnetic field [see Eq. (2)]. Therefore it is natural to expect that the all-optical magnetization reversal, being a result of the interplay between these two effects, should possess a high sensitivity to the parameters of the laser pulse. We have already shown in Sec. IV A that the laser fluence is one of the crucial parameters for the all-optical reversal,

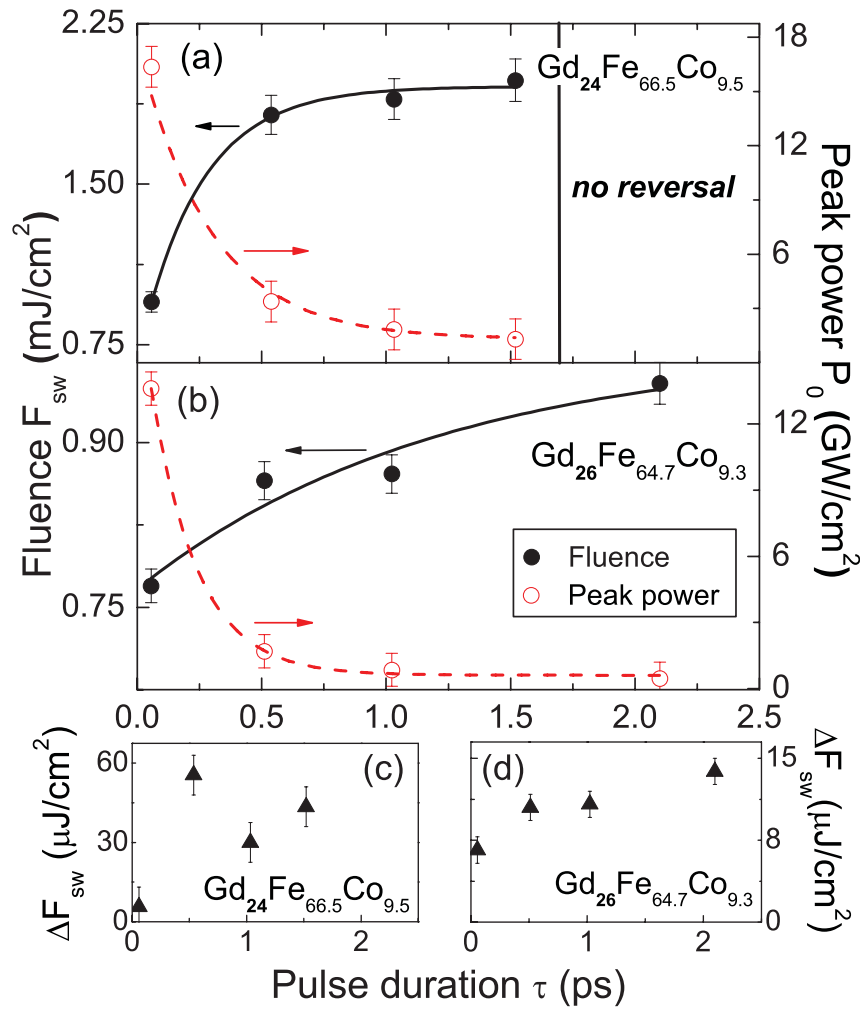

FIG. 8. (Color online) (a) and (b) The laser pulse fluence $F_{\text {sw }}$ and peak power $P_{0}$ at the center of the reversal window and (c) and (d) the width of the reversal window $\Delta F_{\mathrm{sw}}$ vs the duration of the laser pulse $\tau$ as obtained for the samples $\mathrm{Gd}_{24} \mathrm{FeCo}(\mathrm{a})$ and (c) and $\mathrm{Gd}_{26} \mathrm{FeCo}(\mathrm{b})$ and (d) from the fluence dependencies of the switchability measured for different laser pulse durations. Note that in the sample $\mathrm{Gd}_{24} \mathrm{FeCo}$, after the action of the laser pulses with duration $\tau>1.7 \mathrm{ps}$, no reversal was observed. Lines are guides for the eye.

because it defines the electronic temperature increase and, consequently, the degree of demagnetization. Moreover, our calculations suggest that the reversal should be feasible with picosecond laser pulses as well. In order to explore further how the all-optical helicity-dependent reversal process depends on the laser pulse parameters, we studied the reversal for the case of laser pulses of various durations.

\section{Experiment}

In order to investigate experimentally how the change of the laser pulse duration influences the reversal process, we measured the all-optical switchability $\mathcal{P}\left(\sigma^{+}\right)-\mathcal{P}\left(\sigma^{-}\right)$ as a function of the laser pulse fluence for different laser pulse durations. The results for the samples $\mathrm{Gd}_{24} \mathrm{FeCo}$ and $\mathrm{Gd}_{26} \mathrm{FeCo}$ are shown in Fig. 8. In Figs. 8(a) and 8(b), we have plotted the dependence of the laser pulse fluence $F_{\text {sw }}$ and the laser peak power $P_{0}$, which corresponds to $100 \%$ switchability, on the laser pulse duration. The width of the reversal window $\Delta F_{\mathrm{sw}}$ as a functions of the laser pulse duration is shown in Figs. 8(c) and 8(d). As one can see, the reversal window is getting wider as the laser pulse duration increases. Such a behavior is in qualitative agreement with the phase diagram $W-\tau_{\text {OM }}$ [see Fig. 6(a)]. Our experimental data also show that the all-optical switching of the magnetization requires more laser pulse fluence as the pulse duration increases [see 


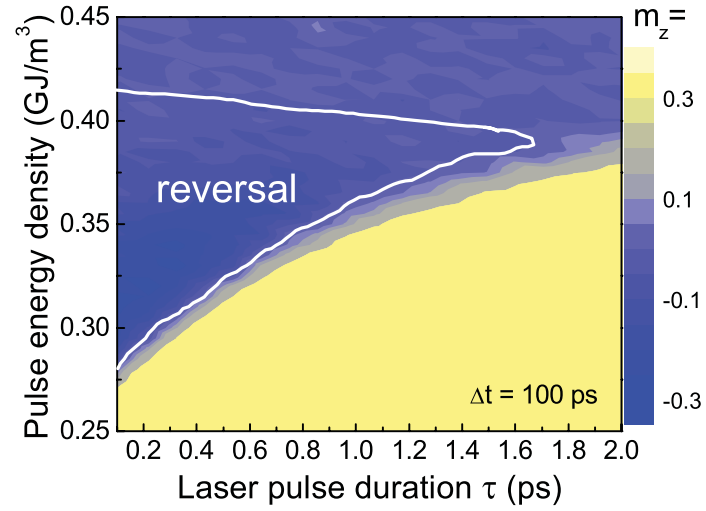

FIG. 9. (Color online) Calculated phase diagram showing the magnetization of the $(30 \mathrm{~nm})^{3}$ element at $100 \mathrm{ps}$ after the action of a laser pulse of energy density $W$ and duration $\tau$. The parameters of the optomagnetic fields are scaled with the parameters of the laser pulse as discussed in Sec. III. The color code for the $m_{\mathrm{z}}$ values is given on the right hand side of the figure. The initial value of $m_{\mathrm{z}}$ is +1 . The solid line separates the region where $m_{z}<0$, i.e., where the probability of the reversal is $>0$.

Figs. 8(a) and 8(b)]. Note that the overall increase of the required laser pulse fluence $F_{\mathrm{sw}}$ with the pulse duration is rather small. As a result, the corresponding peak power $P_{0}$ required for the reversal decreases with an increase of the laser pulse duration. The effects of the laser pulse duration on the reversal are qualitatively the same in $\mathrm{Gd}_{24} \mathrm{FeCo}$ and $\mathrm{Gd}_{26} \mathrm{FeCo}$ alloys. However, in the case of $\mathrm{Gd}_{24} \mathrm{FeCo}$, the all-optical reversal is observed only when the laser pulse duration is shorter than $1700 \mathrm{fs}$. For longer pulses we have observed either no effect (in the case of low pump fluence) or the formation of a multidomain state (in the case of high pump pulse fluence). In the sample $\mathrm{Gd}_{26} \mathrm{FeCo}$, the reversal is observed in the whole range of the studied pulse durations up to $2100 \mathrm{fs}$.

\section{Modeling}

It is natural to expect that an increase of the laser pulse duration will result in an increase of the duration of the optomagnetic field (see Fig. 3). The former, however, also affects the temporal profile of the electronic temperature. This is illustrated in Figs. 3(b) and 3(c), which show the dynamics of the electronic temperature calculated from the two-temperature model for laser pulse durations 100 and $2000 \mathrm{fs}$. To understand the observed dependence of the magnetization reversal on the laser pulse duration, we simulated the dynamics of the magnetization of a $(30 \mathrm{~nm})^{3}$ sample in a single-macrospin approximation. We took into account the scaling of the optomagnetic field and the change of the electronic temperature $T_{\mathrm{el}}$ profile with the change of the laser pulse duration [see Eq. (2)]. Figure 9 shows the magnetic state of the sample 100 ps after the action of the laser pulse with energy density $W$ and duration $\tau$.

As one can see from the presented phase diagram, there is a range of laser pulse durations and fluences, where the probability for the all-optical reversal of the magnetization is nonzero. Similar to the experiment (see Fig. 8), the magnetization reversal by a longer laser pulse requires a higher fluence. Again, similar to the experiment [see Figs. 8(a) and 8(b)], this means that longer pulses can reverse the magnetization with lower peak power $P_{0}$. As it follows from Eq. (3), the peak power of the laser pulse defines the amplitude of the effective field [see Eq. (3)]. Thus, from our experimental and simulated results, we can conclude that, when the effective light-induced field $\mathbf{H}_{\mathrm{OM}}$ has a longer duration, the amplitude of this field can be lower than for the case of shorter pulses. This observation might seem to be counterintuitive. However, the demagnetization dynamics, in contrast to the effective field generation, is determined by the laser pulse fluence rather than by its peak power [see Eq. (6)]. Therefore an enhancement of the fluence $F_{\mathrm{sw}}$ means that the highly nonequilibrium state is reached earlier, which compensates for a lower amplitude of the effective field.

Our calculations show (see Fig. 9) that the width of the reversal window decreases as the pulse duration increases and after certain pulse duration, all-optical reversal cannot be realized. This agrees partly with our experimental data that show the disappearance of the all-optical reversal in sample $\mathrm{Gd}_{24} \mathrm{FeCo}$ for pulses longer than 1700 fs [see Fig. 8(a)]. Moreover, the disappearance of the magnetization reversal for laser pulses with longer duration agrees well with the fact that ultrafast demagnetization cannot be realized when the laser pulse duration becomes too long..$^{5-61}$ We would like to note, however, that the proposed model is genuinely not capable of accounting for the difference between the magnetization reversal by longer pulses in the samples with different concentrations of Gd ions [see Figs. 8(a) and 8(b)], as it treats the medium as a ferromagnetic ensemble of spins.

As it was discussed in Sec. II, the GdFeCo alloys with different Gd concentrations possess different magnetic properties, such as compensation temperature and coercivity. The difference in the all-optical reversal process revealed in the samples with different Gd contents suggests, that the actual magnetic structure plays an important role in the laser-induced magnetic dynamics. The model of the all-optical reversal in a ferrimagnetic medium is still a challenge. Nevertheless, here, we experimentally investigated the effect of the ferrimagnetic structure of the GdFeCo alloys on the speed and efficiency of the all-optical helicity-dependent magnetization reversal.

Concluding the discussion of the parameters of the laser pulse, we would like to add that, as we discovered experimentally, the all-optical reversal can be also realized with elliptically polarized 100 fs laser pulses. Surprisingly, the ellipticity and, consequently, the parameter $\sigma$ in Eq. (3) could be varied in a rather wide range. This accentuates the fact that the question about the origin and realistic calculations of the effective optomagnetic field remains unanswered.

\section{Helicity-dependent all-optical reversal in various ferrimagnetic $\mathrm{Gd}_{x} \mathrm{Fe}_{90-x} \mathrm{Co}_{10}$ alloys}

\section{Efficiency of the helicity-dependent reversal}

If one compares the results of the action of a laser pulse of the same fluence, duration and polarization on samples with different concentration of $\mathrm{Gd}$ ions being at the same equilibrium temperature, a striking difference is clearly seen. Figure 10(a) shows that at at room temperature at a pump fluence $F=3.14 \mathrm{~mJ} / \mathrm{cm}^{2}$ the all-optical magnetization 

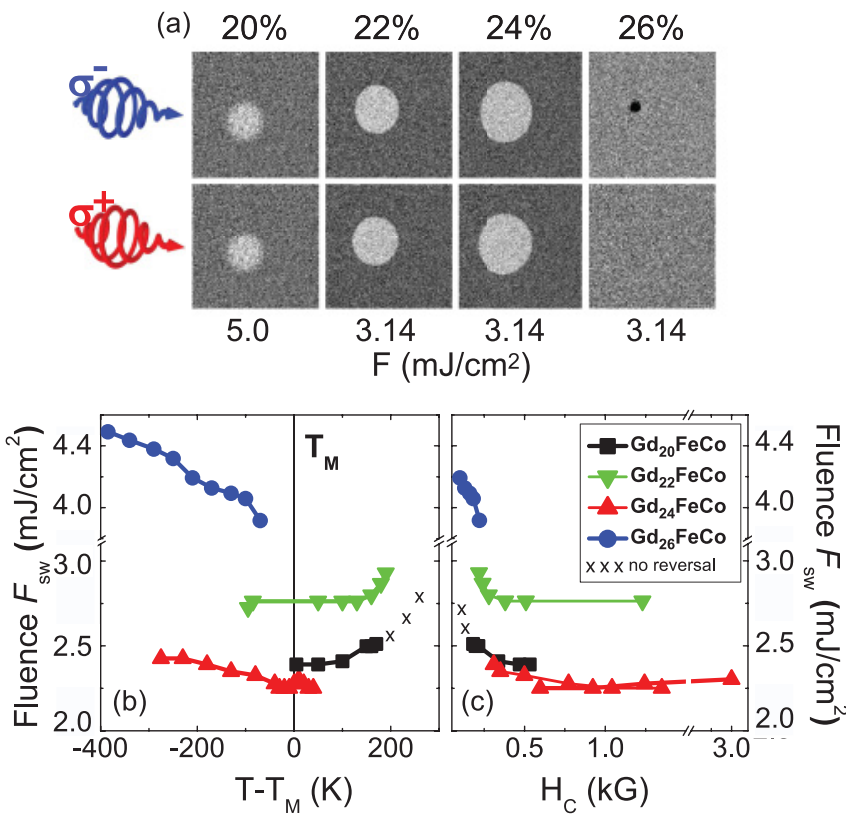

FIG. 10. (Color online) (a) Steady-state magneto-optical images of the samples $\mathrm{Gd}_{x} \mathrm{FeCo}, x=20 \%, 22 \%, 24 \%$, and $26 \%$ obtained after the action of a single 100-fs circularly-polarized laser pulse. The fluence of the laser pulse was $3.14 \mathrm{~mJ} / \mathrm{cm}^{2}$ for all samples except for $\mathrm{Gd}_{20} \mathrm{FeCo}$, where no effect was observed for such a fluence. Before the irradiation, all samples were saturated by applying an external magnetic field pulse of the same direction. Because of the magneto-optical imaging, the orientation of the FeCo sublattice is visualized. Thus, black and white areas correspond to the orientation of the FeCo-sublattice magnetization "up" and "down." It is oriented differently for the sample $\mathrm{Gd}_{26} \mathrm{FeCo}$, because for this sample $T_{\text {comp }}$ is above the room temperature, in contrast to other samples. Consequently, the FeCo sublattice is antiparallel to the magnetic field at the room temperature. Thus, in all shown cases, the initial orientation of the total bulk magnetization is the same. (b) The laser pulse fluence required for the all-optical reversal $F_{\mathrm{sw}}$ as a function of the relative temperature $T-T_{\mathrm{M}}$ for the same samples. The pulse duration in these measurements was $\tau=100 \mathrm{fs}$. The value of $T_{\mathrm{M}}$ at $T \approx 0 \mathrm{~K}$ for the sample $\mathrm{Gd}_{20} \mathrm{FeCo}$ was obtained from the extrapolation of $T_{\mathrm{M}}$ vs $x$ dependence [see inset in Fig. 1(b)]. Crosses indicate the absence of the all-optical reversal for the sample $\mathrm{Gd}_{20} \mathrm{FeCo}$. (c) $F_{\text {sw }}$ plotted vs the coercive field $H_{\mathrm{c}}$ as obtained from Figs. 10(b) and 1(b).

reversal is observed only in the sample $\mathrm{Gd}_{26} \mathrm{FeCo}$, while in the samples $\mathrm{Gd}_{22(24)} \mathrm{FeCo}$, a laser pulse of the same fluence leads to a polarization-independent reversal (see range III in Fig. 4). All-optical helicity-dependent reversal in these samples was also observed but at somewhat lower pump fluences. Surprisingly, in the sample $\mathrm{Gd}_{20} \mathrm{FeCo}$, the action of a single pulse of any polarization results only in the formation of a multidomain state. No all-optical reversal was observed in this sample at room temperature.

The main difference between these samples is the compensation temperature $T_{\mathrm{M}}$ [see inset in Fig. 1(b)]. We have studied the all-optical reversal in these samples at different temperatures. As a quantitative parameter, describing the all-optical reversal, we have chosen the fluence required for the all-optical reversal $F_{\text {sw }}$, falling approximately in the center of the reversal window. As a reference parameter, we also measured the minimum fluence required for the formation of a multidomain final state $F_{\mathrm{md}}$ (range IV in Fig. 4). This study reveals plenty of differences between the samples. In $\mathrm{Gd}_{22} \mathrm{FeCo}, F_{\mathrm{sw}}$ drops as the temperature decreases (similar dependence of $F_{\text {sw }}$ on temperature in the $\mathrm{Gd}_{22} \mathrm{FeCo}$ alloy was reported in Ref. 28). In the sample $\mathrm{Gd}_{20} \mathrm{FeCo}$, which possessed no reversal at room temperature, a decrease of the temperature below $T=200 \mathrm{~K}$ leads to the appearance of all-optical reversal. In contrast, in $\mathrm{Gd}_{24} \mathrm{FeCo}$ and $\mathrm{Gd}_{26} \mathrm{FeCo}$ samples, a temperature decrease had an opposite effect, leading to an increase of $F_{\mathrm{sw}}$. The dependence of $F_{\mathrm{md}}$ on temperature for all samples showed the same trend. At lower temperatures, higher fluences $F_{\mathrm{md}}$ were required for the formation of the multidomain state.

This diverse behavior of the reversal in the studied samples becomes unified if one plots the minimum fluence $F_{\text {sw }}$ required for the all-optical reversal as a function of the difference between the temperature of the sample $T$ and its compensation temperature $T_{\mathrm{M}}$, as shown in Fig. 10(b). From this figure, one can see that for all studied samples, $F_{\mathrm{sw}}$ shows a minimum near the compensation point (at $T-T_{\mathrm{M}}=0$ ). The larger the deviation of the sample temperature from the compensation temperature, the higher the fluence required for the switching.

Note that the linear reversal implies that the fluence of the laser pulse is sufficient to increase the electronic temperature from its equilibrium value up to $T_{\mathrm{el}}^{*}$. Consequently, one can expect that $F_{\text {sw }}$ should be higher when the equilibrium electron temperature, i.e., sample temperature, decreases. The experimental data, however, show that this condition is satisfied only below $T_{\mathrm{M}} \cdot{ }^{58}$ The observed dependence on temperature shows that the ferrimagnetic properties of the medium, which have not been taken into account in our model, strongly affect the reversal process.

When considering the dependence of the fluence required for the reversal on the sample temperature, it is worth noting that the latter affects coercivity among other properties, [see Fig. 1(b)]. In Fig. 10(c), we plotted $F_{\text {sw }}$ obtained from Fig. 10(b) versus the coercive field $H_{c}$. Although the values of $F_{\mathrm{sw}}$ differ for these samples, there is a clear trend in the $F_{\text {sw }}$ versus $H_{\mathrm{c}}$ dependence observed for all samples. More specifically, when the coercive field is high, the fluence $F_{\text {sw }}$ is weakly dependent on the coercive field. In turn, when the coercive field is lower $F_{\text {sw }}$ starts to increase. Furthermore, no reversal is observed when the coercivity is low. It is known that the coercivity determines, in particular, the minimum stable size of a domain that can exist in a medium. Hence, as a result of this, one of the possible reasons for the observed disappearance of the reversal in the samples with low coercivity is that the domain created by the laser pulse of a given spot size cannot be sustained in the medium. We, however, would like to stress that no dependence of the domain size on the temperature and the sample composition was observed. This suggests that the size of the written domain is mostly determined by the area within the laser spot where the fluence satisfies the conditions for the all-optical reversal.

Therefore the study of the efficiency of the all-optical helicity-dependent reversal clearly shows that choosing temperature in the vicinity of the compensation temperature $T_{\mathrm{M}}$ is very important for the reversal process. A lower pump fluence 


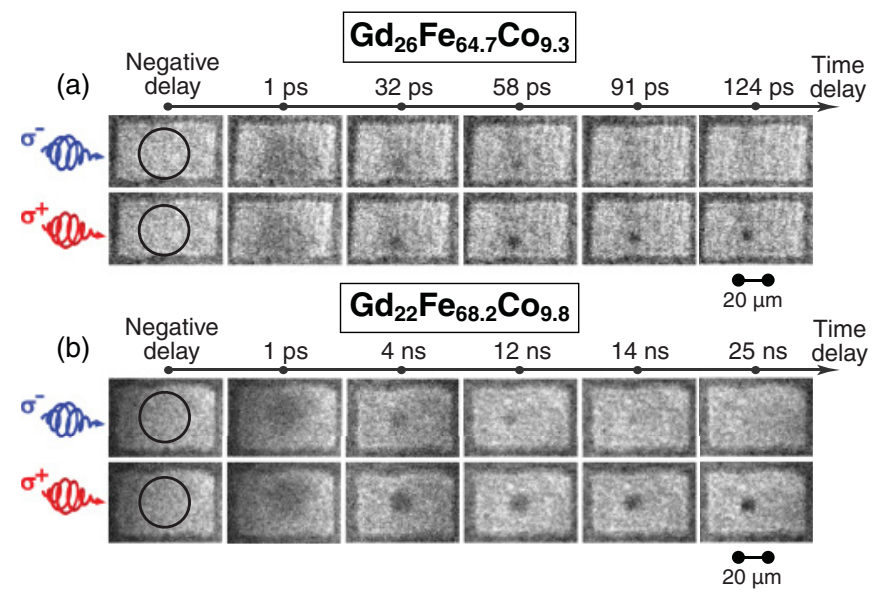

FIG. 11. (Color online) Single-shot magneto-optical images of $\mathrm{Gd}_{x} \mathrm{FeCo}$ for (a) $x=22 \%$ and (b) $x=26 \%$ at room temperature obtained at different time delays after excitation with a single $100-\mathrm{fs}$ circularly polarized laser pulses.

is required to switch magnetization near $T_{\mathrm{M}}$, while far away of the compensation point all-optical magnetization reversal may become not feasible.

\section{Speed of the all-optical reversal in GdFeCo alloys}

It can be shown that along with the efficiency of the all-optical reversal, the switching time appears to be strongly affected by the composition of the GdFeCo alloy. Figure 11 shows the evolution of the magnetic state during the reversal process in the samples $\mathrm{Gd}_{22} \mathrm{FeCo}$ and $\mathrm{Gd}_{26} \mathrm{FeCo}$ at ambient temperature. As one can see, the switching time for $\mathrm{Gd}_{26} \mathrm{FeCo}$ is around $100 \mathrm{ps}$ and comparable with the time obtained for $\mathrm{Gd}_{24} \mathrm{FeCo}$. However, the magnetization switching in $\mathrm{Gd}_{22} \mathrm{FeCo}$ happens on a much longer time scale of $16 \mathrm{~ns}$.

In order to investigate this further, we studied the speed of the reversal as a function of temperature in these three alloys. The write-read time $\tau_{\mathrm{w}-r}$ [as defined in Fig. 7(b)] is plotted in Fig. 12 as a function of the difference between the sample temperature and the compensation point $T-T_{\mathrm{M}}$. Figure 12 shows that the reversal appears to be the fastest for temperatures below $T_{\mathrm{M}}$. In particular, by decreasing the temperature of $\mathrm{Gd}_{22} \mathrm{FeCo}$ one can tune $\tau_{\mathrm{w}-r}$ from $16 \mathrm{~ns}$ at room temperature down to $30 \mathrm{ps}$ at $10 \mathrm{~K}\left(T-T_{\mathrm{M}}=-90 \mathrm{~K}\right)$.

As we have shown in Sec. IV B, the all-optical helicitydependent magnetization reversal is a linear reversal, occurring when the sample is brought to a strongly nonequilibrium state with no net magnetization. This is followed by a relaxation of the sample to an equilibrium monodomain state. Importantly, it is the speed of the latter process that determines the switching time $\tau_{\mathrm{w}-r}$. Obviously, the relaxation to the equilibrium state includes, first of all, a slow heat diffusion from the photoexcited spot, which is typically of the order of nanoseconds. As a result, the magnetization reaches only $80 \%$ of its equilibrium value after $\tau_{\mathrm{w}-r}$ [see Fig. 7(b)]. Since this heat diffusion is determined by heat conductivity of the layers comprising the multilayered structures, there is no reason to expect this time to vary much as a function of composition and temperature.

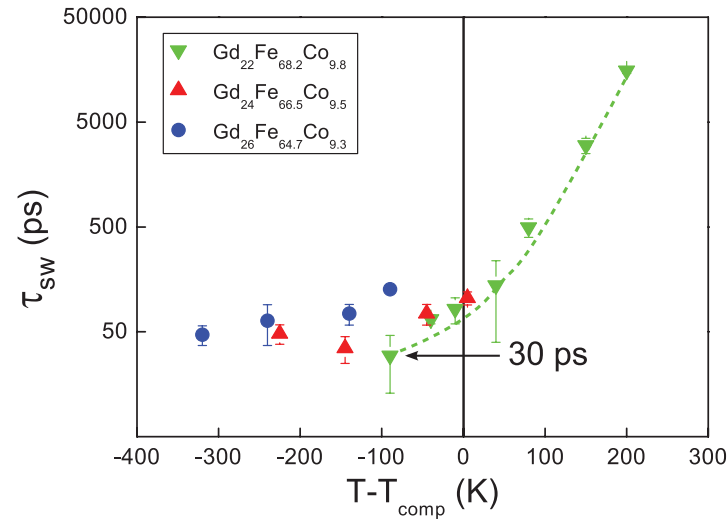

FIG. 12. (Color online) Write-read time $\tau_{\mathrm{w}-r}$ vs relative temperature $T-T_{\mathrm{M}}$ for $\mathrm{Gd}_{22} \mathrm{Fe}_{68.2} \mathrm{Co}_{9.8}\left(T_{\mathrm{M}}=100 \mathrm{~K}\right), \mathrm{Gd}_{24} \mathrm{Fe}_{66.5} \mathrm{Co}_{9.5}$ $\left(T_{\mathrm{M}}=280 \mathrm{~K}\right)$, and $\mathrm{Gd}_{26} \mathrm{Fe}_{64.7} \mathrm{Co}_{9.3}\left(T_{\mathrm{M}}=390 \mathrm{~K}\right){ }^{26}$ We achieved magnetization reversal within $30 \mathrm{ps}$ for $\mathrm{Gd}_{22} \mathrm{Fe}_{68.2} \mathrm{Co}_{9.8}$ at $10 \mathrm{~K}$. The dash line is a guide to the eye. Note that the angular momentum compensation point $T_{\mathrm{A}}$ is $\sim 50 \mathrm{~K}$ above $T_{\mathrm{M}} \cdot{ }^{33}$

Second, the write-read time is dependent on time, which is needed for lattice to equilibrate. According to the twotemperature model [see Eq. (6)], this process is determined by the characteristic time $\tau_{\text {th }}$ and by the amount of energy pumped into the system. We have no arguments to assume that the time $\tau_{\text {th }}$ would be significantly different for different samples. The energy load required for the all-optical reversal, in turn, differs for different samples and different initial temperatures [see Fig. 10(b)]. However, the studies of a magnetization recovery after excitation with the sub-picosecond laser pulse $\mathrm{e}^{57}$ have shown that recovery time is determined predominantly by the degree to which the magnetization was destroyed. Moreover, no correlation between an increase of the pump fluence and the write-read time is observed for the samples with equilibrium temperatures below the compensation point. Instead, our experimental data (see Fig. 12) show that neither absolute temperature of the sample nor the sample composition alone determine the write-read time. $\tau_{\mathrm{w}-r}$ only slightly varies for any sample with equilibrium temperature $T_{0}$ being below $T_{\mathrm{M}}$, and increases drastically as $T_{0}$ starts to exceed $T_{\mathrm{M}}$. This suggests that the ferrimagnetic properties have to be taken into account in order to explain the variations of the write-read times.

One of the possible explanations for this behavior relies on the fact that the formation of the monodomain final includes, alone with the relaxation processes, the domain wall motion. This conclusion can be reached from rather qualitative considerations. Note that the ultrashort laser pulse pushes the medium into a strongly nonequilibrium but magnetic state with no net magnetization. ${ }^{57}$ Since the state is magnetic and no net magnetization is observed, it should be characterized by spin correlations on the length-scale smaller than the wavelength of light. In fact, this is a multidomain state. A transition from a multidomain to a single-domain state must occur via the process of domain wall motion, growth of favorable domains and disappearance of unfavorable ones. The speed of the latter process, naturally, depends on the mobility of the domain walls, which in $\mathrm{GeFeCo}$ alloys is strongly temperature dependent. ${ }^{62-64}$ Moreover, the speed is very high in the vicinity 
of the angular momentum compensation temperature $T_{\mathrm{A}}$. When a sample being below $T_{\mathrm{A}}$ is subjected to a circularly polarized laser pulse, the laser excited area always contains domains brought close to $T_{\mathrm{A}}$ where the domain wall mobility is the largest. Then, it is the mobility at $T_{\mathrm{A}}$ that dominates the averaged domain walls speed in the photoexcited area and, thus, determines the write-read time. Above $T_{\mathrm{A}}$, alloptical magnetization reversal can still be realized but the write-read time increases with temperature, as the optical excitation brings the system even further from $T_{\mathrm{A}}$ (see Fig. 12).

\section{CONCLUSIONS AND OUTLOOK}

Combining the experimental technique of single-shot time-resolved magneto-optical microscopy with multiscale modeling of the nonequilibrium spin dynamics, we have revealed a path for the reversal of the magnetization by a single circularly polarized laser pulse. In particular, we have demonstrated experimentally that in order to reverse the magnetization, the laser pulse should bring the spin system within 1 ps into a strongly nonequilibrium state characterized by a nearly quenched net magnetization. Multiscale modeling of the dynamics of the spin system at this state shows that the simultaneous action of a strong and short heat pulse and a pulse of magnetic field favors the magnetization to follow a so-called linear reversal path, governed by longitudinal relaxation process. Experimentally, such a two-fold action can be obtained using circularly-polarized laser pulses.

By examining, experimentally and computationally, the reversal process for various laser pulses we have shown that the helicity-dependent magnetization switching occurs in a narrow range of laser pulse fluences. Below this range, the conditions for linear reversal are not satisfied, as the latter can occur only when the magnetization is nearly quenched. ${ }^{27}$ Above this range, the degree of disorder brought to the spin system is too high and, thus the strength and duration of the effective light-induced magnetic field are not sufficient for the reversal.

We have found that the all-optical reversal is feasible for a rather wide range of laser pulse durations up to $2100 \mathrm{fs}$. However, our experimental data and the proposed model suggest that there is an upper limit for the laser pulse duration required for the all-optical reversal. Based on our model, we expect that there should also be a lower limit. This, however, still has to be verified in future experiments with ultrashort laser pulses.

The feasibility and the characteristics of the all-optical reversal process are also dependent on the magnetic properties of the GdFeCo alloys. Although the all-optical reversal can be observed above and below the compensation temperature of ferrimagnetic $\mathrm{GdFeCo}$ alloys, in the vicinity of $T_{\mathrm{M}}$, the reversal requires less laser pulse fluence. Moreover, the all-optical reversal disappears if the sample temperature is too high above the compensation temperature. These results suggest that the ferrimagnetic properties of the samples play a crucial role in the all-optical magnetization reversal. Consequently, development of comprehensive approaches for simulations of nonequilibrium spin dynamics in multisublattice magnets are urgently required.
We also demonstrated that the all-optical helicitydependent magnetization reversal time is determined by the relaxation process from the strongly nonequilibrium state and by the mobility of the domain walls, in particular. This leads to a drastic decrease of the reversal time in the samples below their compensation temperature. Therefore we expect that ferrimagnetic media with compensation points above room temperature are promising candidates for the realization of fast all-optical magnetic recording. We would like to mention that the achieved write-read time of $30 \mathrm{ps}$ in one of the GdFeCo alloys (see Fig. 12) is the shortest write-read time demonstrated for magnetic recording so far. Moreover, our experimental studies and modeling suggest that this time can be reduced further by a proper tailoring of the magnetic and magneto-optical properties of a recording medium.

As a concluding remark, we would like to stress that our study of the all-optical reversal in RE-TM alloys highlights the role of ferrimagnetism in the process. Not only does the ferrimagnetism affect the write-read time, the experimental findings suggest that the ferrimagnetic structure of $\mathrm{GdFeCo}$ alloys also influences the features of linear reversal at a ultrashort time scales. A further understanding how such a complex magnetic structure influences the ultrafast laserinduced spin dynamics will be a challenging task for both theoreticians and experimentalists. Development of models and theories capable of accounting for realistic interactions in a magnetic medium is required. For further experimental studies of the ultrafast dynamics in ferrimagnets, experiments with higher spatial and temporal resolution and with element specificity (to distinguish, for example, $\mathrm{Gd}$ from $\mathrm{Fe}$ and $\mathrm{Co}$ ) will be desirable. ${ }^{29,65-67}$ We believe that such studies will be profitable for the whole area of ultrafast laser-induced spin dynamics.

Note added. Very recently, Steil et al. ${ }^{68}$ reported the results of a study of the all-optical reversal in $\mathrm{Gd}_{26} \mathrm{Fe}_{64.7} \mathrm{Co}_{9.3}$ with laser pulses of various durations. The trends revealed in this publication agree well with the findings reported here.

\section{ACKNOWLEDGMENTS}

The authors thank I. Radu for the insightful discussions and help with samples characterization, S. Kaneko for the help with some experiments, C. D. Stanciu for the useful discussions at the early stages of the study, A. F. van Etteger, and A. J. Toonen for the technical support.

This research has received funding from De Nederlandse Organisatie voor Wetenschappelijk Onderzoek (NWO), de Stichting voor Fundamenteel Onderzoek der Materie (FOM), the Dutch Nanotechnology Initiative NanoNed, European Commission (EC) 7th Framework Programme under grants No. NMP3-SL-2008-214469 (UltraMagnetron) and No. 214810 (FANTOMAS) as well as the European Research Council (ERC) grant agreement No. N257280 (Femtomagnetism). The work in Konstanz was supported by the Ministry of Science, Research and Arts of Baden-Württemberg via the Center for Applied Photonics. A.M.K. acknowledges support from the Russian Foundation for Basic Research (grant No. 10-02-01008-a). 
*kalashnikova@mail.ioffe.ru

†a.kimel@science.ru.nl

${ }^{1}$ J. Stöhr and H. C. Siegmann, Magnetism: From Fundamentals to Nanoscale Dynamics, Springer Series in Solid-State Sciences (Springer, Berlin, 2006).

${ }^{2}$ Th. Gerrits, H. A. M. van den Berg, J. Hohlfeld, L. Bär, and Th. Rasing, Nature (London) 418, 509 (2002).

${ }^{3}$ I. Tudosa, C. Stamm, A. B. Kashuba, F. King, H. C. Siegmann, J. Stohr, G. Ju, B. Lu, and D. Weller, Nature (London) 428, 831 (2004).

${ }^{4}$ A. Kashuba, Phys. Rev. Lett. 96, 047601 (2006).

${ }^{5}$ E. Beaurepaire, J.-C. Merle, A. Daunois, and J.-Y. Bigot, Phys. Rev. Lett. 76, 4250 (1996).

${ }^{6}$ B. Koopmans, M. van Kampen, J. T. Kohlhepp, and W. J. M. de Jonge, Phys. Rev. Lett. 85, 844 (2000).

${ }^{7}$ F. Dalla Longa, J. T. Kohlhepp, W. J. M. de Jonge, and B. Koopmans, Phys. Rev. B 75, 224431 (2007).

${ }^{8}$ C. Boeglin, E. Beaurepaire, V. Halté, V. López-Flores, C. Stamm, N. Pontius, H. A. Dürr, and J.-Y. Bigot, Nature (London) 465, 458 (2010).

${ }^{9}$ M. Battiato, K. Carva, and P. M. Oppeneer, Phys. Rev. Lett. 105, 027203 (2010).

${ }^{10}$ M. S. Si and G. P. Zhang, J. Phys. Condens. Matter 22, 076005 (2010).

${ }^{11}$ C. Stamm, T. Kachel, N. Pontius, R. Mitzner, T. Quast, K. Holldack, S. Khan, C. Lupulescu, E. F. Aziz, M. Wietstruk, H. A. Dürr, and W. Eberhardt, Nat. Mater. 6, 740 (2007).

${ }^{12}$ B. Koopmans, J. E. M. Haverkort, W. J. M. de Jonge, and G. Karczewski, J. Appl. Phys. 85, 6763 (1999).

${ }^{13}$ J.-Y. Bigot, L. Guidoni, E. Beaurepaire, and P. N. Saeta, Phys. Rev. Lett. 93, 077401 (2004).

${ }^{14}$ E. Beaurepaire, G. M. Turner, S. M. Harrel, M. C. Beard, J.-Y. Bigot, and C. A. Schmuttenmaer, Appl. Phys. Lett. 84, 3465 (2004).

${ }^{15}$ J. Güdde, U. Conrad, V. Jahnke, J. Hohlfeld, and E. Matthias, Phys. Rev. B 59, R6608 (1999).

${ }^{16}$ M. Aeschlimann, M. Bauer, S. Pawlik, W. Weber, R. Burgermeister, D. Oberli, and H. C. Siegmann, Phys. Rev. Lett. 79, 5158 (1997).

${ }^{17}$ A. Scholl, L. Baumgarten, R. Jacquemin, and W. Eberhardt, Phys. Rev. Lett. 79, 5146 (1997).

${ }^{18}$ Ł. Cywiński and L. J. Sham, Phys. Rev. B 76, 045205 (2007).

${ }^{19}$ N. Kazantseva, D. Hinzke, U. Nowak, R. W. Chantrell, U. Atxitia, and O. Chubykalo-Fesenko, Phys. Rev. B 77, 184428 (2008).

${ }^{20}$ G. Lefkidis, G. P. Zhang, and W. Hübner, Phys. Rev. Lett. 103, 217401 (2009).

${ }^{21}$ B. Koopmans, G. Malinowski, F. Dalla Longa, D. Steiauf, M. Fähnle, T. Roth, M. Cinchetti, and M. Aeschlimann, Nature Mater. 9, 259 (2010).

${ }^{22}$ G. P. Zhang, Y. Bai, and T. F. George, Phys. Rev. B 80, 214415 (2009).

${ }^{23}$ V. N. Gridnev, Phys. Rev. B 77, 094426 (2008).

${ }^{24}$ A. Kirilyuk, A. V. Kimel, and Th. Rasing, Rev. Mod. Phys. 82, 2731 (2010).

${ }^{25}$ C. D. Stanciu, F. Hansteen, A. V. Kimel, A. Kirilyuk, A. Tsukamoto, A. Itoh, and Th. Rasing, Phys. Rev. Lett. 99, 047601 (2007).

${ }^{26}$ K. Vahaplar, A. M. Kalashnikova, A. V. Kimel, D. Hinzke, U. Nowak, R. W. Chantrell, A. Tsukamoto, A. Itoh, A. Kirilyuk, and Th. Rasing, Phys. Rev. Lett. 103, 117201 (2009).

${ }^{27}$ N. Kazantseva, D. Hinzke, R. W. Chantrell, and U. Nowak, Europhys. Lett. 86, 27006 (2009).
${ }^{28}$ J. Hohlfeld, C. D. Stanciu, and A. Rebei, Appl. Phys. Lett. 94, 152504 (2009).

${ }^{29}$ I. Radu, K. Vahaplar, C. Stamm, T. Kachel, N. Pontius, H. A. Dürr, T. A. Ostler, J. Barker, R. F. L. Evans, R. W. Chantrell, A. Tsukamoto, A. Itoh, A. Kirilyuk, Th. Rasing, and A. V. Kimel, Nature (London) 472, 205 (2011).

${ }^{30}$ M. Mansuripur, The Physical Principles of Magneto-Optical Recording (Cambridge University Press, Cambridge, 1995).

${ }^{31}$ X. Jiang, L. Gao, J. Z. Sun, and S. S. P. Parkin, Phys. Rev. Lett. 97, 217202 (2006)

${ }^{32}$ Y. Mimura, N. Imamura, T. Kobayashi, A. Okada, and Y. Kushiro, J. Appl. Phys. 49, 1208 (1978).

${ }^{33}$ C. D. Stanciu, A. V. Kimel, F. Hansteen, A. Tsukamoto, A. Itoh, A. Kiriliyuk, and T. Rasing, Phys. Rev. B 73, 220402 (2006).

${ }^{34}$ H. Tsujimoto, M. Shouji, A. Saito, S. Matsushita, and Y. Sakurai, J. Magn. Soc. Jpn. 7, 119 (1983).

${ }^{35}$ N. Endo, S. Masui, T. Kobayashi, S. Tsunashima, and S. Uchiyama, J. Magn. Soc. Jpn. 8, 101 (1984).

${ }^{36}$ S. P. Pershan, Phys. Rev. 130, 919 (1963).

${ }^{37}$ M. V. Chetkin, A. P. Kuzmenko, S. N. Gadetskii, V. N. Filatov, and A. I. Akhutkina, Pis'ma Zh. Eksp. Teor. Fiz. 37, 223 (1983) [JETP Lett. 37, 264 (1983)].

${ }^{38}$ M. V. Chetkin, S. N. Gadetskii, and A. I. Akhutkina, Pis'ma Zh. Eksp. Teor. Fiz. 35, 373 (1982) [JETP Lett. 35, 459 (1982)].

${ }^{39}$ W. R. Hendren, R. Atkinson, R. J. Pollard, I. W. Salter, C. D. Wright, W. W. Clegg, and D. F. L. Jenkins, J. Phys. Condens. Matter 15, 1461 (2006).

${ }^{40}$ F. H. Liu, M. D. Schultz, and M. H. Kryder, IEEE Trans. Magn. 26, 1340 (1990).

${ }^{41}$ M. Elazar, M. Sahaf, L. Szapiro, D. Cheskis, and S. Bar-Ad, Opt. Lett. 33, 2734 (2008).

${ }^{42}$ A. V. Kimel, A. Kirilyuk, P. A. Usachev, R. P. Pisarev, A. M. Balbashov, and Th. Rasing, Nature (London) 435, 655 (2005).

${ }^{43}$ R. Hertel, J. Magn. Magn. Mater. 303, L1 (2006).

${ }^{44}$ G. P. Zhang, W. Hubner, G. Lefkidis, Y. H. Bai, and T. F. George, N. Phys. 5, 499 (2009).

${ }^{45}$ L. P. Pitaevskii, Zh. Eksp. Teor. Fiz. 39, 1450 (1960) [Sov. Phys. JETP 12, 1008 (1961)].

${ }^{46}$ P. S. Pershan, J. P. van der Ziel, and L. D. Malmstrom, Phys. Rev. 143, 574 (1966).

${ }^{47}$ K. Carva, M. Battiato, and P. M. Oppeneer, N. Phys. 7, 665 (2011).

${ }^{48}$ P. M. Oppeneer and A. Liebsch, J. Phys. Condens. Matter 16, 5519 (2004).

${ }^{49}$ H. Petek and S. Ogawa, Prog. Surf. Sci. 56, 239 (1997).

${ }^{50}$ V. V. Kruglyak, R. J. Hicken, M. Ali, B. J. Hickey, A. T. G. Pym, and B. K. Tanner, Phys. Rev. B 71, 233104 (2005).

${ }^{51}$ H.-L. Zhang, Y.-Zh. Wang, X.-J. Chen, J. Magn. Magn. Mater. 321, L73 (2009).

${ }^{52}$ T. Yoshino, J. Magn. Magn. Mater. 323, 2531 (2011).

${ }^{53}$ D. J. Hilton, R. D. Averitt, C. A. Meserole, G. L. Fisher, D. J. Funk, J. D. Thompson, and A. J. Taylor, Opt. Lett. 29, 1805 (2004).

${ }^{54}$ M. I. Kaganov, I. M. Lifshitz, and L. V. Tanatarov, Zh. Eksp. Teor. Fiz. 31, 232 (1957) [Sov. Phys. JETP 4, 173 (1957)].

${ }^{55} \mathrm{G}$. Zhang, W. Hübner, E. Beaurepaire, and J.-Y. Bigot, Spin Dynamics in Confined Magnetic Structures I, Topics in Applied Physics Vol. 83 (Springer, New York, 2002). 
${ }^{56}$ D. A. Garanin, Phys. Rev. B 55, 3050 (1997).

${ }^{57}$ N. Kazantseva, U. Nowak, R. W. Chantrell, J. Hohlfeld, and A. Rebei, Europhys. Lett. 81, 27004 (2008).

${ }^{58} \mathrm{We}$ note that the decrease of $F_{\mathrm{sw}}$ with temperature in $\mathrm{Gd}_{22} \mathrm{FeCo}$, reported in Ref. 28, lead the authors to the conclusion that the all-optical reversal has an athermal character and does not involve the process of ultrafast laser-induced demagnetization. Our results clearly show that the observed behavior is only typical for samples above the compensation temperature $T_{\mathrm{M}}$.

${ }^{59}$ M. B. Agranat, S. I. Ashitkov, A. B. Granovsky, and G. I. Rukman, Zh. Eksp. Teor. Fiz. 86, 1376 (1984) [Sov. Phys. JETP 59, 804 (1984)].

${ }^{60}$ M. B. Agranat, S. I. Ashitkov, A. V. Kirillin, V. E. Fortov, S. I. Anisimov, A. B. Granovskii, and P. S. Kondratenko, Pis'ma Zh. Eksp. Teor. Fiz. 67, 904 (1998) [JETP Lett. 67, 953 (1998)].
${ }^{61}$ A. Vaterlaus, D. Guarisco, M. Lutz, M. Aeshlimann, M. Stampanoni, and F. Meyer, J. Appl. Phys. 67, 5661 (1990).

${ }^{62}$ T. Miyama, S. Matsushita, H. Tsujimoto, and Y. Sakurai, IEEE Trans. Magn. 14, 728 (1978).

${ }^{63}$ R. S. Weng and M. H. A. Kryder, IEEE Trans. Magn. 29, 2177 (1993).

${ }^{64}$ V. V. Randoshkin, V. A. Polezhaev, N. N. Sysoev, and Y. N. Sazhin, Phys. Solid State 45, 513 (2003).

${ }^{65}$ S. Eisebitt, J. Luning, W. F. Schlotter, M. Lorgen, O. Hellwig, W. Eberhardt, and J. Stohr, Nature (London) 432, 885 (2004).

${ }^{66}$ B. Koopmans, Nat. Mater. 6, 715 (2007).

${ }^{67}$ C. Gutt et al., Phys. Rev. B 81, 100401(R) (2010).

${ }^{68}$ D. Steil, S. Alebrand, A. Hassdenteufel, M. Cinchetti, and M. Aeschlimann, Phys. Rev. B 84, 224408 (2011). 